\title{
A weak version of bivariate lack of memory property
}

\author{
Nikolai Kolev and Jayme Pinto \\ University of São Paulo
}

\begin{abstract}
We suggest a modification of the classical Marshall-Olkin's bivariate exponential distribution considering a possibility of a singularity contribution along arbitrary line through the origin. It serves as a base of a new weaker version of the bivariate lack of memory property, which might be both "aging" and "non-aging" depending on the additional inclination parameter. The corresponding copula is obtained and we establish its disagreement with Lancaster's phenomena. Characterizations and properties of the novel bivariate memory-less notion are obtained and its applications are discussed. We characterize associated weak multivariate version. The weak bivariate lack of memory property implies restrictions on the marginal distributions. Starting from pre-specified marginals we propose a procedure to build bivariate distributions possessing a weak bivariate lack of memory property and illustrate it by examples. We complement the methodology with closure properties of the new class. We finish with a discussion and suggest several related problems for future research.
\end{abstract}

\section{Introduction and preliminaries}

The classical bivariate lack of memory property (BLMP) has at least half century history since the paper of Marshall and Olkin (1967). Many textbooks use as a base and give a special attention to the BLMP and related bivariate exponential distribution exhibiting singularity along the main diagonal in $R_{+}^{2}=[0, \infty) \times[0, \infty)$, see Barlow and Proschan (1981), Singpurwalla (2006), Balakrishnan and Lai (2009), Gupta, Zeung and Hu (2010), Cherubini, Durante and Mulinacci (2015), McNeil, Frey and Embrechts (2015) among others. More than 2000 articles complement and extend Marshall-Olkin's bivariate exponential distribution, justifying advantages in analysis of various data sets from engineering, medicine, insurance, finance, biology, etc. Let us mention two very recent contributions only: Lin, Lai and Govindaraju (2016) and Brigo, Mai and Scherer (2016). The reader would find in Lin, Lai and Govindaraju (2016) interesting and new investigations regarding the correlation coefficient and dependence structure of bivariate Marshall-Olkin's exponential distribution. On the other side, Brigo, Mai and Scherer (2016) offer a new characterization of Marshall-Olkin multivariate law (e.g., all sub-vectors of

Key words and phrases. Bivariate Marshall-Olkin's exponential distribution, copula, closure properties, failure rate, lack of memory property, non-aging and aging performance, reliability, stochastic representation, singularity.

Received July 2016; accepted August 2017. 
associated survival indicators are continuous-time Markov chains) with a direct impact to overcome practical limitations for the modeling of high-dimensional default times. In this article, we suggest one more modification of the BLMP, allowing singularity along the arbitrary line through the origin $(0,0)$.

To proceed, let us begin with the classical bivariate Marshall-Olkin (MO) shock model specified by the stochastic representation

$$
\left(X_{1}, X_{2}\right)=\left(\min \left(T_{1}, T_{3}\right), \min \left(T_{2}, T_{3}\right)\right),
$$

where non-negative continuous random variables $T_{1}$ and $T_{2}$ identify the occurrence of independent "individual shocks" affecting two devices and $T_{3}$ is their "common shock". The random vector $\left(X_{1}, X_{2}\right)$ presents the joint distribution of both lifetimes.

Specifically, let $\left(X_{1}, X_{2}\right)$ be a continuous non-negative random vector defined by its joint survival function $S_{X_{1}, X_{2}}\left(x_{1}, x_{2}\right)=P\left(X_{1}>x_{1}, X_{2}>x_{2}\right)$ for all $x_{1}, x_{2} \geq 0$. If the shocks are governed by independent homogeneous Poisson processes, then $T_{i}$ 's in (1.1) are exponentially distributed with parameters $\gamma_{i}>0, i=1,2,3$, and we obtain the $M O$ bivariate exponential distribution given by

$$
S_{X_{1}, X_{2}}\left(x_{1}, x_{2}\right)=\exp \left\{-\gamma_{1} x_{1}-\gamma_{2} x_{2}-\gamma_{3} \max \left(x_{1}, x_{2}\right)\right\}, \quad x_{1}, x_{2} \geq 0,
$$

see Marshall and Olkin (1967). Due to the "common shock" distinguished by $T_{3}$ in (1.1), this distribution owns a singular component along the line $\left\{x_{1}=x_{2}\right\}$ in $R_{+}^{2}$, with a weight $P\left(X_{1}=X_{2}\right)=\frac{\gamma_{3}}{\gamma_{1}+\gamma_{2}+\gamma_{3}}>0$, as shows the scatterplot displayed on Figure 1(a). Hence, the MO bivariate exponential distribution is not absolutely continuous, that is, it does not have a probability density with respect to the twodimensional Lebesgue measure.

To give a probability formalization of model (1.1), consider a system composed by two items, to be denoted by 1 and 2 . We associate with each item $j, j=1,2$, a Bernoulli random variable $Z_{j}$, indicating whether the item is operational $\left(Z_{j}=1\right)$ or failed $\left(Z_{j}=0\right)$. The bivariate Bernoulli random vector $\left(Z_{1}, Z_{2}\right)$ represents the state of the system. It is specified in terms of MO construction (1.1). The vector $\left(X_{1}, X_{2}\right)$ exhibits the latent state of the system, since the MO model (1.2) is defined in terms of vector $\left(T_{1}, T_{2}, T_{3}\right)$ of latent variables that identify independent exponential shock times. Each shock takes down a given subset of items $(\{1\},\{2\}$ or \{both 1 and 2$\}$ ) and occurs at an exponential time with constant rates $\gamma_{1}, \gamma_{2}$ and $\gamma_{3}$, respectively.

The distribution (1.2) is the only solution with exponential marginals of functional equation

$S_{X_{1}, X_{2}}\left(x_{1}+t, x_{2}+t\right)=S_{X_{1}, X_{2}}\left(x_{1}, x_{2}\right) S_{X_{1}, X_{2}}(t, t) \quad$ for all $x_{1}, x_{2}, t \geq 0$,

characterizing the BLMP. Relation (1.3) tells us that, independent of $t$, the BLMP preserves the joint distribution of both $\left(X_{1}, X_{2}\right)$ and its residual lifetime vector $\mathbf{X}_{t}=\left[\left(X_{1}-t, X_{2}-t\right) \mid X_{1}>t, X_{2}>t\right]$ and their marginal distributions, therefore. 
(a)

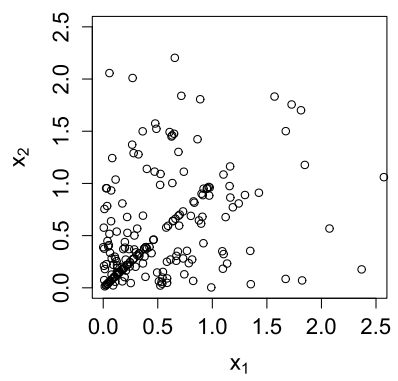

(b)

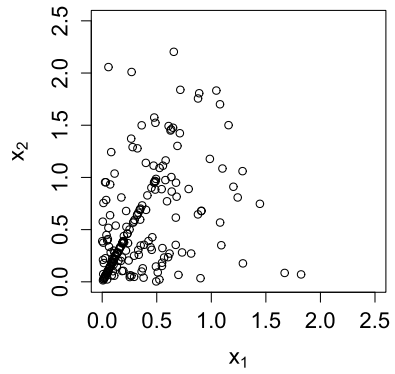

Figure 1 Plots with singularity along the lines $x_{1}=x_{2}$ and $x_{1}=0.5 x_{2}$. (a) MO bivariate exponential distribution (1.2): $\omega=1, \gamma_{1}=\gamma_{2}=\gamma_{3}=1$; (b) modified MO biv. exp. distribution (1.4): $\omega=0.5, \lambda_{1}=\lambda_{2}=\lambda_{3}=1$.

The MO bivariate exponential distribution (1.2) has exponential marginals with parameters $\gamma_{1}+\gamma_{3}$ and $\gamma_{2}+\gamma_{3}$ and hence, constant marginal failure (hazard) rates. This restricts its usefulness for practical needs. As a response, other solutions of (1.3) with non-exponential marginals have been introduced. Let us mention Block and Basu (1974), Proschan and Sullo (1974), Friday and Patil (1977). An important contribution to the bivariate lack of memory notion is offered by Kulkarni (2006) who suggested a class of bivariate distributions possessing BLMP specified by (1.3), having increasing or/and decreasing marginal failure rates, but they should satisfy a set of restrictions. Many other authors are cited by Balakrishnan and Lai (2009) in their Chapter 10.

The stochastic relation (1.1) is widely used in literature. For example, Li and Pellerey (2011) launched the Generalized MO model considering non-exponential independent random variables $T_{i}$ in (1.1), $i=1,2,3$. The corresponding joint distributions do not possess BLMP, that is, are "aging". As a further step, Pinto and Kolev (2015) introduced the Extended MO model assuming dependence between variables $T_{1}$ and $T_{2}$, but keeping $T_{3}$ independent of them. The motivation is that the individual shocks might be dependent if the items share a common environment. In this case however, BLMP may be fulfilled or not depending on parameters of joint distribution of $\left(T_{1}, T_{2}\right)$ and distribution of $T_{3}$.

For a specific data set, it may happen that a cluster of the scatter plot points is concentrated on an arbitrary continuous curve in $R_{+}^{2}$, or along arbitrary line through the origin $(0,0)$, in particular, as shown on Figure 1(b). One would be unsuccessful to model adequately such bivariate data with distributions generated by stochastic representation (1.1).

A possible alternative to (1.2) is the modified MO bivariate exponential distribution defined as follows

$$
S_{X_{1}, X_{2}}\left(x_{1}, x_{2}\right)=\exp \left\{-\lambda_{1} x_{1}-\lambda_{2} x_{2}-\lambda_{3} \max \left(x_{1}, \omega x_{2}\right)\right\}, \quad x_{1}, x_{2} \geq 0,
$$


where $\omega>0$. At least one of parameters $\lambda_{i}, i=1,2,3$, should be positive, otherwise one would have a degenerate distribution. The marginals in (1.4) are exponentially distributed with parameters $\lambda_{1}+\lambda_{3}$ and $\lambda_{2}+\omega \lambda_{3}$. One can recognize that there exists a positive mass concentrated along the line $L_{\omega}:\left\{x_{1}=\omega x_{2}\right\}$ through the origin $(0,0)$ in $R_{+}^{2}$. It can be verified that the singularity contribution is $P\left(X_{1}=\omega X_{2}\right)=\frac{\omega \lambda_{3}}{\omega \lambda_{1}+\lambda_{2}+\omega \lambda_{3}}>0$. Obviously, $X_{1}$ and $X_{2}$ are independent if $\lambda_{3}=0$.

Statistical inference related to the modified MO bivariate exponential distribution (1.4) is presented by Okyere (2007) who provided maximum likelihood estimation of parameters when $\omega$ is known, along with several asymptotic results.

One can check that (1.4) is a solution of the functional equation

$$
S_{X_{1}, X_{2}}\left(x_{1}+\omega t, x_{2}+t\right)=S_{X_{1}, X_{2}}\left(x_{1}, x_{2}\right) S_{X_{1}, X_{2}}(\omega t, t),
$$

for all $x_{1}, x_{2}, t \geq 0$ and $\omega>0$.

The BLMP functional equation (1.3) can be obtained from (1.5) when $\omega=1$. In other words, the functional equation (1.5) exhibits a weak version of BLMP. Thus, we are ready to introduce the following definition.

Definition 1.1. A bivariate non-negative continuous distribution whose joint survival function satisfies the functional equation (1.5) possesses the weak bivariate lack of memory property, or shortly W-BLMP.

Our aim in this article is to investigate the W-BLMP defined by functional equation (1.5). In Section 2, we discuss structural properties of modified MO bivariate exponential distribution (1.4) and compare it with the classical one (1.2). We obtain the corresponding modified MO survival copula and discuss its features. In Section 3, we characterize the W-BLMP and present its multivariate version. In Section 4, we recognize that only certain marginal distributions are allowed for bivariate continuous distributions satisfying (1.5). The corresponding restrictions in terms of marginal densities and failure rates are derived. We suggest a procedure to construct bivariate distributions belonging to the W-BLMP class starting from given (pre-specified) marginal distributions. We support the methodology by typical examples and complement it by closure properties of the W-BLMP class. We finish with a discussion and pose several possible problems for further investigation.

\section{Modified Marshall-Olkin bivariate exponential distribution}

In fact, the modified MO bivariate exponential distribution (1.4) has been introduced by Esary and Marshall (1974) in their Example 2.3 by the following expression

$$
S_{X_{1}, X_{2}}\left(x_{1}, x_{2}\right)=\exp \left\{-\xi_{1} x_{1}-\xi_{2} x_{2}-\max \left(\xi_{3} x_{1}, \xi_{4} x_{2}\right)\right\}, \quad x_{1}, x_{2} \geq 0,
$$


where $\xi_{1}+\xi_{3}>0, \xi_{2}+\xi_{4}>0$ and $\xi_{i} \geq 0, i=1,2,3,4$. This distribution has been used as a base and motivation to define a novel class of bivariate distributions exhibiting exponential scaled minima. One can recognize the associated relation in the second proof of Theorem 1 . If substitute $\lambda_{i}=\xi_{i}, i=1,2,3$ and $\omega=\frac{\xi_{4}}{\xi_{3}}$ in last relation one will get (1.4).

Let us note that the modified MO bivariate exponential distribution (1.4) is positive quadrant dependent since $S_{X_{1}, X_{2}}\left(x_{1}, x_{2}\right) \geq S_{X_{1}}\left(x_{1}\right) S_{X_{2}}\left(x_{2}\right)$ for all $x_{1}, x_{2} \geq 0$.

We will discuss in the next properties of the modified MO bivariate exponential distribution (1.4) and then we compare it with MO's distribution (1.2). We obtain the survival copula corresponding to (1.4) as well and outline the disagreement with Lancaster's phenomena.

\subsection{Basic properties}

The modified MO bivariate exponential distribution (1.4) can be generated by the following two stochastic representations:

1. Let $\left(Y_{1}, Y_{2}\right)$ follow the MO bivariate exponential distribution (1.2) obtained by stochastic relation (1.1). The distribution (1.4) results if substitute $X_{1}=Y_{1}$ and $X_{2}=\frac{Y_{2}}{\omega}$ with $\omega>0$ in (1.1). In this case, $\lambda_{1}=\gamma_{1}, \lambda_{2}=\omega \gamma_{2}$ and $\lambda_{3}=\gamma_{3}$.

2. The joint survival function in (1.4) is a consequence of stochastic representation

$$
\left(X_{1}, X_{2}\right)=\left[\min \left(T_{1}, T_{3}\right), \min \left(T_{2}, \frac{T_{3}}{\omega}\right)\right],
$$

where $\omega>0$ and the random variables $T_{i}$ are independent and exponentially distributed with parameters $\lambda_{i}>0$, respectively, $i=1,2,3$.

Really, for all $x_{1}, x_{2} \geq 0$ and $\omega>0$ we have

$$
\begin{aligned}
P\left(X_{1}>x_{1}, X_{2}>x_{2}\right) & =P\left(\min \left(T_{1}, T_{3}\right)>x_{1}, \min \left(T_{2}, \frac{T_{3}}{\omega}\right)>x_{2}\right) \\
& =P\left(T_{1}>x_{1}, T_{2}>x_{2}, T_{3}>\max \left(x_{1}, \omega x_{2}\right)\right) .
\end{aligned}
$$

Taking into account that $T_{i}$ are independent and exponentially distributed with parameters $\lambda_{i}, i=1,2,3$, we obtain the joint survival function given by (1.4).

Remark 2.1 (Reliability interpretation of (2.1)). Denote by $X_{i}$ the lifetime of a component $i, i=1,2$. If $0<\omega<1$, relation (2.1) tells us that a common "fatal shock" destroys immediately the first component and has a delayed effect on the second one. For example, assume that only one of two identical devices operating in the same factory is equipped with a electricity generator protecting against possible blackout. The stochastic relation (2.1) indicates that the lifetime represented by $X_{2}$ will be greater than the lifetime $X_{1}$ of unprotected device if a common "fatal shock" (governed by a homogeneous Poisson process) occurs. 
Therefore, an important characteristic of distribution (1.4) resulting from (2.1), is that it permits a "late" failure of one component when a "fatal shock" strikes both components (as against to the MO models generated by (1.1) where both components fail simultaneously if occurs a common "fatal shock" distinguished by the random variable $T_{3}$ ).

On the other side, the physical meaning of equation (1.5) is the following: the conditional probability that both components survive an "additional" time $\left(x_{1}, x_{2}\right)$ after surviving $(\omega t, t)$ time units is supposed to be equal to unconditional probability of surviving to time $\left(x_{1}, x_{2}\right)$, i.e.,

$$
P\left(X_{1}>x_{1}+\omega t, X_{2}>x_{2}+t \mid X_{1}>\omega t, X_{2}>t\right)=P\left(X_{1}>x_{1}, X_{2}>x_{2}\right) .
$$

In terms of joint survival function the last relation can be equivalently written by functional equation (1.5). It says that the $\left(X_{1}, X_{2}\right)$ and corresponding $\omega$-type residual lifetime vector, to denote it by

$$
\mathbf{X}_{\omega, t}=\left[\left(X_{1}-\omega t, X_{2}-t\right) \mid X_{1}>\omega t, X_{2}>t\right],
$$

should have the same joint distribution, independent of $t>0$ for all $x_{1}, x_{2} \geq 0$ and $\omega>0$. The identical memory-less property is valid for marginal distributions of $\left(X_{1}, X_{2}\right)$ and $\mathbf{X}_{\omega, t}$. Obviously, if $\omega=1$, then $\mathbf{X}_{\omega, t}$ transforms into the residual lifetime vector $\mathbf{X}_{t}$ associated to the BLMP.

A basic issue in the bivariate data analysis is to study the monotonicity (e.g., increasing/decreasing performance) of the survival function of residual lifetime vector $\mathbf{X}_{t}$ corresponding to the vector $\left(X_{1}, X_{2}\right)$. In fact, the main interest is to establish when the ratio $Q_{1}\left(x_{1}, x_{2}, t\right)=\frac{S_{X_{1}, X_{2}}\left(x_{1}+t, x_{2}+t\right)}{S_{X_{1}, X_{2}}\left(x_{1}, x_{2}\right)}$ is non-decreasing (nonincreasing) in $x_{1}, x_{2} \geq 0$ for all $t \geq 0$, see Barlow and Proschan (1981).

Therefore, an important problem would be to investigate the monotonicity of the $\omega$-type residual lifetime vector $\mathbf{X}_{\omega, t}$ corresponding to the vector $\left(X_{1}, X_{2}\right)$ as well. In this case, the analysis is related to function $Q_{2}\left(x_{1}, x_{2}, t\right)=\frac{S_{X_{1}, X_{2}}\left(x_{1}+\omega t, x_{2}+t\right)}{S_{X_{1}, X_{2}}\left(x_{1}, x_{2}\right)}$, where $\omega>0$.

Under the above notations, we establish relationships between bivariate exponential distributions (1.4) and (1.2) in the next two lemmas.

Lemma 2.1. Let $\left(X_{1}, X_{2}\right)$ follow the modified MO bivariate exponential distribution (1.4) for some $\omega \in(0,1)$. Then

$$
\begin{aligned}
& Q_{1}\left(x_{1}, x_{2}, t\right) \\
& \quad= \begin{cases}S_{X_{1}, X_{2}}(t, t)=S_{X_{3}}\left(a_{1} t\right), & \text { if } x_{1} \geq \omega x_{2}, t \geq 0, \\
S_{X_{3}}\left(h\left(x_{1}, x_{2}, t\right)\right), & \text { if } x_{1}<\omega x_{2}, t>\frac{\omega x_{2}-x_{1}}{1-\omega}, \\
S_{X_{3}}\left(a_{2} t\right), & \text { if } x_{1}<\omega x_{2}, 0 \leq t \leq \frac{\omega x_{2}-x_{1}}{1-\omega},\end{cases}
\end{aligned}
$$


where $S_{X_{3}}$ is the survival function of unit exponential random variable $X_{3}, a_{1}=$ $\lambda_{1}+\lambda_{2}+\lambda_{3}, a_{2}=\lambda_{1}+\lambda_{2}+\omega \lambda_{3}$ and $h\left(x_{1}, x_{2}, t\right)=a_{1} t-\lambda_{3}\left(\omega x_{2}-x_{1}\right) \geq 0$.

Proof. First, note that the ratio $Q_{1}\left(x_{1}, x_{2}, t\right)=\frac{S_{X_{1}, X_{2}}\left(x_{1}+t, x_{2}+t\right)}{S_{X_{1}, X_{2}}\left(x_{1}, x_{2}\right)}$ represents the conditional probability

$$
P_{1}\left(t \mid x_{1}, x_{2}\right)=P\left(X_{1}>x_{1}+t, X_{2}>x_{2}+t \mid X_{1}>x_{1}, X_{2}>x_{2}\right) .
$$

Let $x_{1} \geq \omega x_{2}$. Apply (1.4) with $\omega \in(0,1)$ to get the first expression for $Q_{1}\left(x_{1}, x_{2}, t\right)$ in (2.2).

Now assume that $x_{1}<\omega x_{2}$ and use again (1.4) to obtain

$S_{X_{1}, X_{2}}\left(x_{1}+t, x_{2}+t\right)=\exp \left\{-\lambda_{1}\left(x_{1}+t\right)-\lambda_{2}\left(x_{2}+t\right)-\lambda_{3} \max \left[x_{1}+t, \omega\left(x_{2}+t\right)\right]\right\}$.

If $t>\frac{\omega x_{2}-x_{1}}{1-\omega}$, then $\max \left[x_{1}+t, \omega\left(x_{2}+t\right)\right]=x_{1}+t$ and we arrive to the second expression in (2.2). In this case we have $\exp \left\{-h\left(x_{1}, x_{2}, t\right)\right\} \leq 1$, which ensures that conditional probability $P_{1}\left(t \mid x_{1}, x_{2}\right) \leq 1$.

When $t<\frac{\omega x_{2}-x_{1}}{1-\omega}$ we have $\max \left[x_{1}+t, \omega\left(x_{2}+t\right)\right]=\omega\left(x_{2}+t\right)$ and we obtain the third relation in (2.2).

A graphical illustration of the ratio $\frac{S_{X_{1}, X_{2}}\left(x_{1}+t, x_{2}+t\right)}{S_{X_{1}, X_{2}}\left(x_{1}, x_{2}\right)}$ for fixed $t$ and parameter values $\lambda_{1}=1, \lambda_{2}=1, \lambda_{3}=1, \omega=0.5$ in $(2.2)$ is given on Figure 2 .

In Figure 3, we display the "level curves" of $Q_{1}\left(x_{1}, x_{2}, t\right)$ from Figure 2 by additionally fixing $x_{2}=0.76$ in (2.2). Observe that W-BLMP is represented by BLMP when $x_{1} \geq \omega x_{2}$.

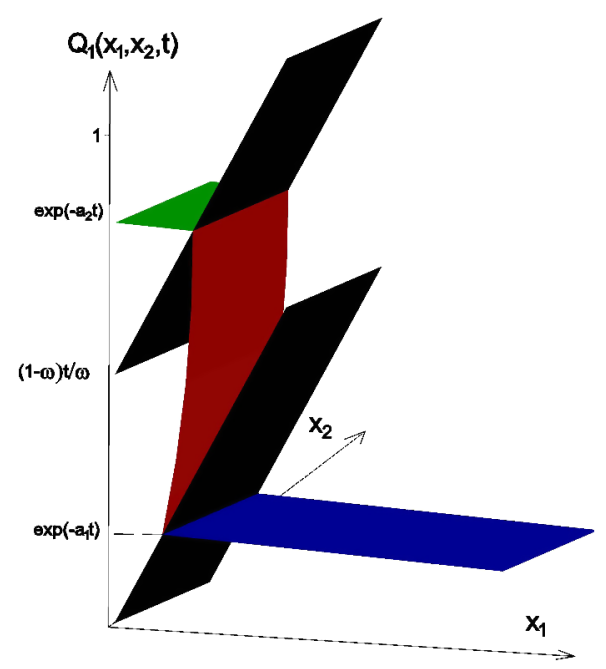

Figure 2 Graph of $Q_{1}\left(x_{1}, x_{2}, t\right)=\frac{S_{X_{1}, X_{2}}\left(x_{1}+t, x_{2}+t\right)}{S_{X_{1}, X_{2}}\left(x_{1}, x_{2}\right)}$ for a fixed $t$ letting $\lambda_{1}=1.0, \lambda_{2}=1.0$, $\lambda_{3}=1.0$ and $\omega=0.5$ in $(2.2)$. 


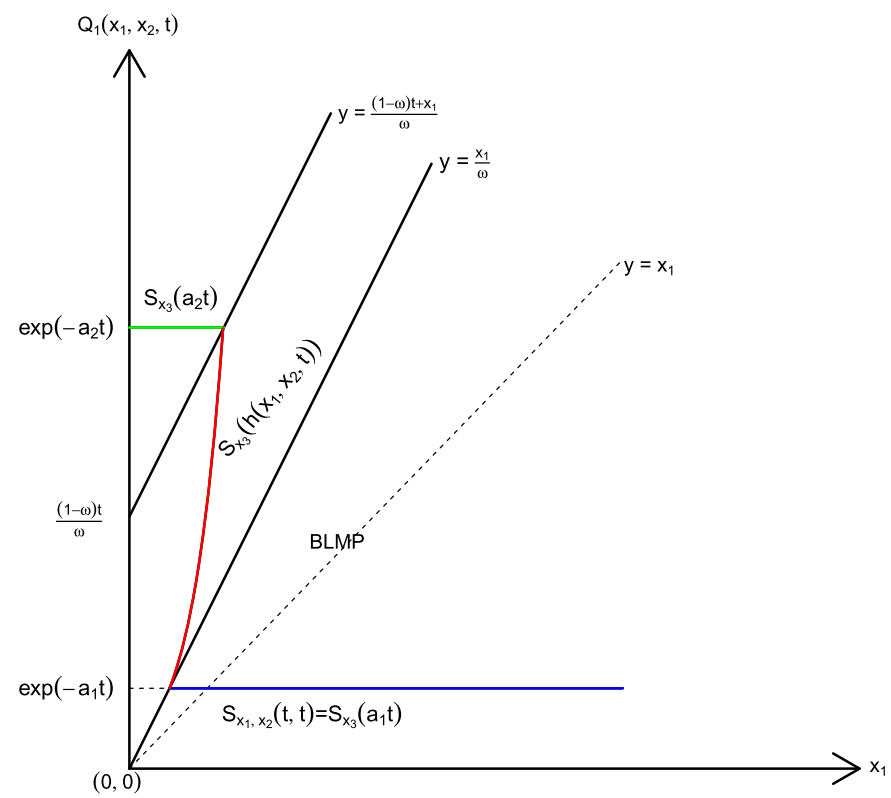

Figure 3 Level curves of $Q_{1}\left(x_{1}, x_{2}, t\right)=\frac{S_{X_{1}, X_{2}}\left(x_{1}+t, x_{2}+t\right)}{S_{X_{1}, X_{2}}\left(x_{1}, x_{2}\right)}$ for fixed $t$, when $x_{2}=0.76, \lambda_{1}=1.0$, $\lambda_{2}=1.0, \lambda_{3}=1.0$ and $\omega=0.5$ in $(2.2)$.

Remark 2.2 (Graphical interpretation of (2.2)). The expressions in Lemma 2.1 can be interpreted as follows.

1. The bivariate distribution (1.4) possesses BLMP below the line $\left\{x_{2}=\frac{x_{1}}{\omega}\right\}$, i.e. the BLMP and W-BLMP worlds are equivalent given that $x_{1} \geq \omega x_{2}$ and $\omega \in$ $(0,1)$.

2. If $x_{1}<\omega x_{2}$ and $t>\frac{\omega x_{2}-x_{1}}{1-\omega}$, then the conditional probability $P_{1}\left(t \mid x_{1}, x_{2}\right)$ exhibits an "aging" behavior in the set $\left\{\left(x_{1}, x_{2}\right) \in R_{+}^{2}: \frac{x_{1}}{\omega}<x_{2}<\frac{x_{1}+(1-\omega) t}{\omega}\right\}$. In this case, we can write

$$
S_{X_{1}, X_{2}}\left(x_{1}+t, x_{2}+t\right)=S_{X_{1}, X_{2}}\left(x_{1}, x_{2}\right) S_{X_{3}}\left(h\left(x_{1}, x_{2}, t\right)\right),
$$

where $X_{3}$ is exponentially distributed with parameter 1 evaluated at

$$
h\left(x_{1}, x_{2}, t\right)=\left(\lambda_{1}+\lambda_{2}+\lambda_{3}\right) t-\lambda_{3}\left(\omega x_{2}-x_{1}\right) \geq 0 .
$$

In this case W-BLMP is "aging".

3. If $x_{1}<\omega x_{2}$ and $t \leq \frac{\omega x_{2}-x_{1}}{1-\omega}$, then $P_{1}\left(t \mid x_{1}, x_{2}\right)$ is independent of $x_{1}$ and $x_{2}$ in the set $\left\{\left(x_{1}, x_{2}\right) \in R_{+}^{2}: \frac{x_{1}+(1-\omega) t}{\omega} \leq x_{2} \leq \exp \left(-a_{2} t\right)\right\}$, that is, W-BLMP exhibits "non-aging" performance as well, but with a lower intensity than the usual one, since $a_{2}<a_{1}$. 
The function $Q_{2}\left(x_{1}, x_{2}, t\right)=\frac{S_{X_{1}, X_{2}}\left(x_{1}+\omega t, x_{2}+t\right)}{S_{X_{1}, X_{2}}\left(x_{1}, x_{2}\right)}$ is analyzed in the following claim. Note that $Q_{2}\left(x_{1}, x_{2}, t\right)$ is equal to the conditional probability

$$
P_{2}\left(t \mid x_{1}, x_{2}\right)=P\left(X_{1}>x_{1}+\omega t, X_{2}>x_{2}+t \mid X_{1}>x_{1}, X_{2}>x_{2}\right) .
$$

Lemma 2.2. Let $\left(X_{1}, X_{2}\right)$ follow the MO bivariate exponential distribution (1.2). If $\omega \in(0,1)$ we have

$$
Q_{2}\left(x_{1}, x_{2}, t\right)= \begin{cases}S_{X_{1}, X_{2}}(\omega t, t)=S_{X_{3}}\left(b_{1} t\right), & \text { if } x_{1} \leq x_{2}, t \geq 0, \\ S_{X_{3}}\left(g\left(x_{1}, x_{2}, t\right)\right), & \text { if } x_{1}>x_{2}, t>\frac{x_{1}-x_{2}}{1-\omega}, \\ S_{X_{3}}\left(b_{2} t\right), & \text { if } x_{1}>x_{2}, 0 \leq t \leq \frac{x_{1}-x_{2}}{1-\omega},\end{cases}
$$

where $S_{X_{3}}$ is the survival function of unit exponential random variable $X_{3}, b_{1}=$ $\gamma_{1} \omega+\gamma_{2}+\gamma_{3}, b_{2}=\gamma_{1} \omega+\gamma_{2}+\gamma_{3} \omega$ and $g\left(x_{1}, x_{2}, t\right)=b_{1} t-\gamma_{3}\left(x_{1}-x_{2}\right) \geq 0$.

Proof. Follows step by step the proof of Lemma 2.1 .

A graphical interpretation of the expressions in Lemma 2.2 is given on Figure 4 for fixed values of the parameters and arguments $x_{2}$ and $t$ in (1.2).

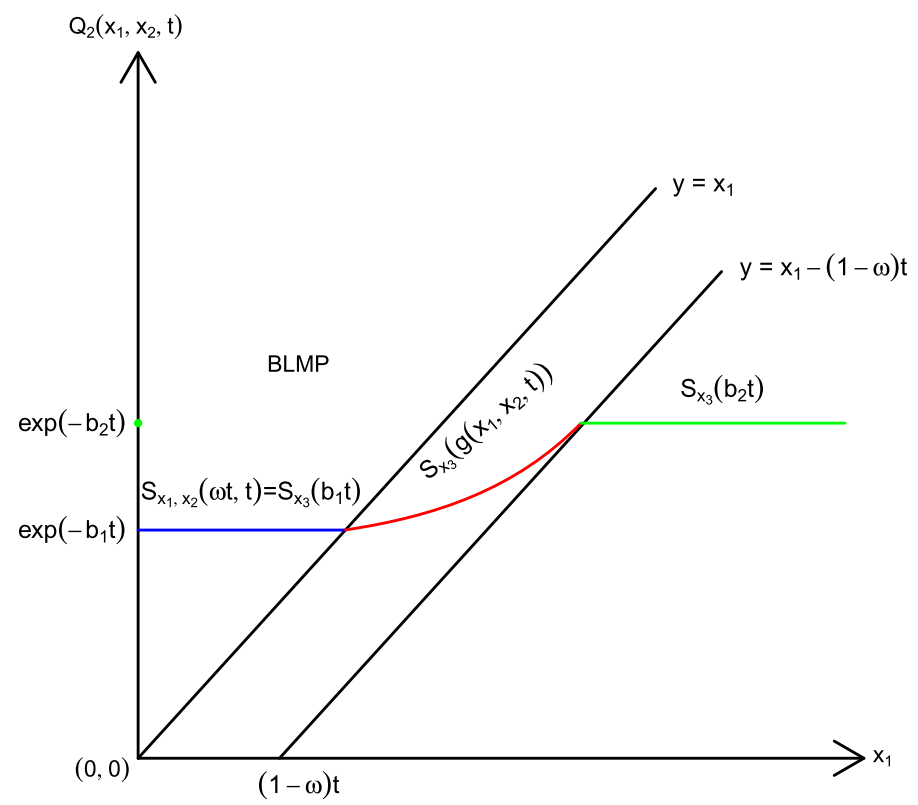

Figure 4 Level curves of $Q_{2}\left(x_{1}, x_{2}, t\right)=\frac{S_{X_{1}, X_{2}}\left(x_{1}+\omega t, x_{2}+t\right)}{S_{X_{1}, X_{2}}\left(x_{1}, x_{2}\right)}$ for fixed arguments $x_{2}=2.0$ and $t$, letting $\gamma_{1}=1, \gamma_{2}=1, \gamma_{3}=1$ and $\omega=0.5$ in $(1.2)$. 
One can observe that the BLMP coincides with the W-BLMP in the set $\left\{x_{2} \geq x_{1}\right\}$ and does not depend on $x_{1}$ and $x_{2}$ when $x_{1} \geq x_{2}-(1-\omega) t$, i.e. being a "non-aging" and again with lower intensity than expected because $b_{1}>b_{2}$. But in the set $\left\{x_{1}<x_{2}<x_{1}+(1-\omega) t\right\}$ the W-BLMP exhibits an "aging" performance.

The expressions in Lemmas 2.1 and 2.2 are valid if $\omega \in(0,1)$. One can obtain alternative formulas for $Q_{1}\left(x_{1}, x_{2}, t\right)$ and $Q_{2}\left(x_{1}, x_{2}, t\right)$ when $\omega>1$.

\subsection{Modified Marshall-Olkin survival copula}

Denote by $\bar{C}_{\mathrm{MO}}\left(u, v ; \sigma_{1}, \sigma_{2}\right)$ the survival copula corresponding to MO bivariate exponential distribution (1.2). It is given by

$$
\bar{C}_{\mathrm{MO}}\left(u, v ; \sigma_{1}, \sigma_{2}\right)=u v \min \left\{u^{-\sigma_{1}}, v^{-\sigma_{2}}\right\}, \quad u, v \in(0,1),
$$

where

$$
\sigma_{1}=\frac{\lambda_{3}}{\lambda_{1}+\lambda_{3}} \quad \text { and } \quad \sigma_{2}=\frac{\lambda_{3}}{\lambda_{2}+\lambda_{3}}, \quad \text { with } \lambda_{i}=\gamma_{i}, i=1,2,3,
$$

see Joe (2015), p. 183.

Now we will obtain the modified MO survival copula corresponding to (1.4). Let $S_{X_{1}, X_{2}}\left(x_{1}, x_{2}\right)$ be given by (1.4). The univariate survival functions are $S_{X_{1}}\left(x_{1}\right)=\exp \left\{-\left(\lambda_{1}+\lambda_{3}\right) x_{1}\right\}$ and $S_{X_{2}}\left(x_{2}\right)=\exp \left\{-\left(\lambda_{2}+\omega \lambda_{3}\right) x_{2}\right\}$. Since $\max \left(x_{1}, \omega x_{2}\right)=x_{1}+\omega x_{2}-\min \left(x_{1}, \omega x_{2}\right)$ we have

$$
\begin{aligned}
S_{X_{1}, X_{2}}\left(x_{1}, x_{2}\right) & =\exp \left\{-\left(\lambda_{1}+\lambda_{3}\right) x_{1}-\left(\lambda_{2}+\omega \lambda_{3}\right) x_{2}+\min \left(x_{1}, \omega x_{2}\right)\right\} \\
& =S_{X_{1}}\left(x_{1}\right) S_{X_{2}}\left(x_{2}\right) \exp \left\{\lambda_{3} \min \left(x_{1}, \omega x_{2}\right)\right\} \\
& =S_{X_{1}}\left(x_{1}\right) S_{X_{2}}\left(x_{2}\right) \min \left[\exp \left\{\lambda_{3} x_{1}\right\}, \exp \left\{\omega \lambda_{3} x_{2}\right\}\right] .
\end{aligned}
$$

Let $u=S_{X_{1}}\left(x_{1}\right)$, that is, $x_{1}=S_{X_{1}}^{-1}(u)$ and therefore $\exp \left\{\lambda_{3} x_{1}\right\}=u^{-\frac{\lambda_{3}}{\lambda_{1}+\lambda_{3}}}$. By analogy, putting $v=S_{X_{2}}\left(x_{2}\right)$ we obtain $\exp \left\{\omega \lambda_{3} x_{2}\right\}=v^{-\frac{\omega \lambda_{3}}{\lambda_{2}+\omega \lambda_{3}}}$. Thus, the modified MO survival copula, to denote it by $\bar{C}_{\mathrm{MMO}}\left(u, v ; \sigma_{1}, \phi_{2}(\omega)\right)$, is given by

$$
\bar{C}_{\mathrm{MMO}}\left(u, v ; \sigma_{1}, \phi_{2}(\omega)\right)=S_{X_{1}, X_{2}}\left(S_{X_{1}}^{-1}(u), S_{X_{2}}^{-1}(v)\right)=u v \min \left\{u^{-\sigma_{1}}, v^{-\phi_{2}(\omega)}\right\},
$$

where $u, v \in(0,1)$ and $\phi_{2}(\omega)=\frac{\omega \lambda_{3}}{\lambda_{2}+\omega \lambda_{3}}$. This expression can be rewritten as

$$
\bar{C}_{\mathrm{MMO}}\left(u, v ; \sigma_{1}, \phi_{2}(\omega)\right)=u^{\sigma_{1}} v^{\phi_{2}(\omega)} \min \left\{u^{1-\sigma_{1}}, v^{1-\phi_{2}(\omega)}\right\} .
$$

On Figure 5 are displayed scatter plots of modified MO survival copula (2.3) with $\lambda_{1}=1.0, \lambda_{2}=0.3$ and $\lambda_{3}=1.0$ for four different values of $\omega$. When $\omega=1$ we recover the MO survival copula $\bar{C}_{\text {MO }}$, see Figure $5(\mathrm{~b})$. One can observe the influence of $\omega$ on the shape of the corresponding singularity line.

Several particular cases and properties of the modified MO survival copula (2.3) are listed below. 
(a)

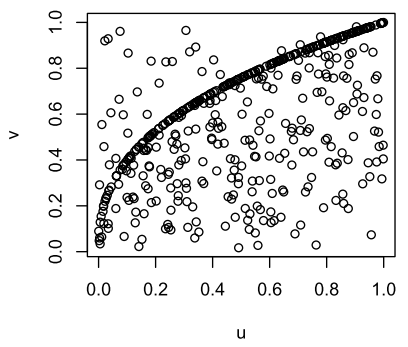

(c)

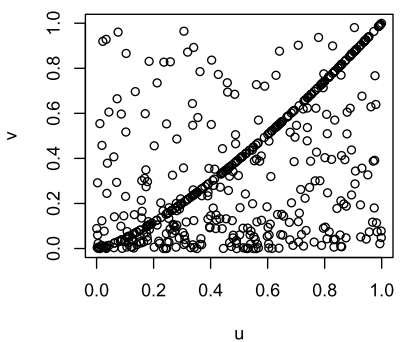

(b)

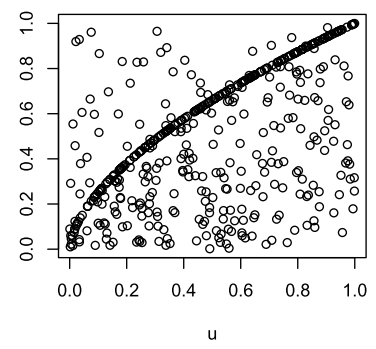

(d)

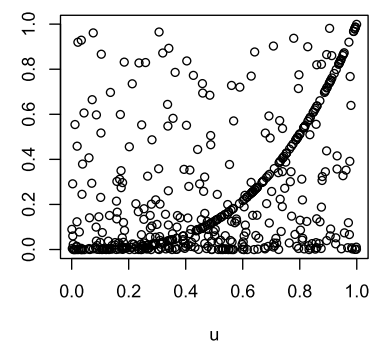

Figure 5 Scatterplots of modified MO copula for $\lambda_{1}=1.0, \lambda_{2}=0.3$ and $\lambda_{3}=1.0$ when (a) $\omega=1.5$; (b) $\omega=1.0$; (c) $\omega=0.5$ and (d) $\omega=0.2$.

- The MO survival copula $\bar{C}_{\mathrm{MO}}\left(u, v ; \sigma_{1}, \sigma_{2}\right)$ results if substitute $\omega=1$ in (2.3).

- If put $\lambda_{2}=\omega \lambda_{2}^{*}$ in (2.3) one will get the MO survival copula with a second parameter $\sigma_{2}^{*}=\frac{\lambda_{3}}{\lambda_{2}^{*}+\lambda_{3}}$, for example, $\bar{C}_{\mathrm{MO}}\left(u, v ; \sigma_{1}, \sigma_{2}^{*}\right)$.

- When $\omega=1$ and $\lambda_{1}=\lambda_{2}$ or $\lambda_{2}=\omega \lambda_{1}$ one obtains the symmetric Cuadras-Auge copula, see Joe (2015), p. 183.

- The lower and upper tail dependence coefficients $\lambda_{L}=\lim _{t \rightarrow 0^{+}} \frac{C(t, t)}{t}$ and $\lambda_{U}=\lim _{t \rightarrow 1^{-}} \frac{\bar{C}(t, t)}{1-t}$ of the modified MO copula are given by $\lambda_{L}=0$ and $\lambda_{U}=\min \left\{\sigma_{1}, \phi_{2}(\omega)\right\}$. Thus, $\bar{C}_{\mathrm{MMO}}\left(u, v ; \sigma_{1}, \phi_{2}(\omega)\right)$ can be used to model the extremal dependence as well.

Remark 2.3 (Power representations of the modified MO copula). First remind that if $C(u, v)$ is a copula, then its power copula $C_{P}(u, v)$ is defined as

$$
C_{P}(u, v)=u^{\eta_{1}} v^{\eta_{2}} C\left(u^{1-\eta_{1}} v^{1-\eta_{2}}\right)
$$

for some additional parameters $\eta_{1}, \eta_{2} \in[0,1]$. The following two relations are valid.

- From representation (2.3) one can recognize that the modified MO copula is, in fact, a power copula corresponding to the upper Frechet bound copula $\min (u, v)$, see Example 1 in Liebscher (2008). 
- The MO survival copula $\bar{C}_{\mathrm{MO}}\left(u, v ; \sigma_{1}, \sigma_{2}\right)$ and its modified MO survival copula $\bar{C}_{\mathrm{MMO}}\left(u, v ; \sigma_{1}, \phi_{2}(\omega)\right)$ are related by equation

$$
\bar{C}_{\mathrm{MMO}}\left(u, v ; \sigma_{1}, \phi_{2}(\omega)\right)=v^{1-\eta} \bar{C}_{\mathrm{MO}}\left(u, v^{\eta} ; \sigma_{1}, \sigma_{2}\right),
$$

where the parameter $\eta=\frac{\omega\left(\lambda_{2}+\lambda_{3}\right)}{\lambda_{2}+\omega \lambda_{3}}$ belongs to the interval $[0,1]$, if and only if $\omega \in[0,1]$.

Therefore, the family of modified MO copulas can be used to model a complementary amount of bivariate asymmetry induced by $\bar{C}_{\mathrm{MO}}\left(u, v ; \sigma_{1}, \sigma_{2}\right)$, see supporting comments in Joe (2015), p. 184. This additional asymmetry does not imply, in general, an increase of extremal dependence governed by the MO survival copula exhibiting upper tail dependence coefficient $\lambda_{U}=\min \left\{\sigma_{1}, \sigma_{2}\right\}$. Depending on parameter values it may happen that $\phi_{2}(\omega)<\sigma_{2}$. As a confirmation, compare the scatterplot (b) on Figure 5 (corresponding to MO survival copula) with others.

The four parameters $\lambda_{1}, \lambda_{2}, \lambda_{3}$ and $\omega$ of modified MO model (1.4) are reduced to three parameters (e.g., $\sigma_{1}$ and $\left.\phi_{2}(\omega)\right)$ of the associated survival copula specified by (2.3). For corresponding Spearman's rho $\rho_{C}=12 \int_{0}^{1} \int_{0}^{1} \bar{C}_{\mathrm{MMO}}(u, v) d u d v-3$, we obtain

$$
\rho_{C}=\frac{3 \sigma_{1} \phi_{2}(\omega)}{2 \sigma_{1}-\sigma_{1} \phi_{2}(\omega)+2 \phi_{2}(\omega)} .
$$

The last expression in terms of $\lambda_{1}, \lambda_{2}, \lambda_{3}$ and $\omega$ can be rewritten as

$$
\rho_{C}=\frac{3 \omega \lambda_{3}}{2\left(\omega \lambda_{1}+\lambda_{2}\right)+3 \omega \lambda_{3}}=\frac{\omega \lambda_{3}}{\frac{2}{3}\left(\omega \lambda_{1}+\lambda_{2}\right)+\omega \lambda_{3}} .
$$

If $S_{X_{1}, X_{2}}\left(x_{1}, x_{2}\right)$ is specified by (1.4), the Pearson correlation coefficient $\rho_{\mathrm{W}-\mathrm{BLMP}}$ is given by

$$
\rho_{\mathrm{W}-\mathrm{BLMP}}=P\left(X_{1}=\omega X_{2}\right)=\frac{\omega \lambda_{3}}{\omega \lambda_{1}+\lambda_{2}+\omega \lambda_{3}},
$$

that is, $\rho_{\mathrm{W}-\mathrm{BLMP}}<\rho_{C}$. Hence, we proved the following statement.

Lemma 2.3. The Pearson's correlation coefficient of the modified MO bivariate exponential distribution (1.4) is less than the Spearman's rho of the associated modified MO copula (2.3), regardless of parameters.

Since a nonlinear transformation of bivariate continuous distribution results in associated copula, Lemma 2.3 implies that distribution (1.4) contradicts the Lancaster's phenomena stating that any nonlinear transformation of variables decreases the correlation in absolute value, see Lancaster (1957).

Note that $\rho_{\mathrm{W}-\mathrm{BLMP}}$ increases as $\lambda_{3}$ increases while keeping $\omega \lambda_{1}+\lambda_{2}$ fixed. Similarly, $\rho_{C}$ decreases when $\omega \lambda_{1}+\lambda_{2}$ increases while keeping $\lambda_{3}$ fixed. When $\omega=1$ one will get the conclusions obtained by Lin, Lai and Govindaraju (2016) for MO distribution (1.2), where complementary properties of dependence structure are obtained. 


\section{Characterizations}

Here we will characterize the W-BLMP offering two alternative proofs. A multivariate version of W-BLMP will be presented as well.

\subsection{Characterization of the weak bivariate lack of memory property}

To proceed, denote by $S_{X}(x)=P(X>x)$ the survival function of continuous random variable $X$. Let us recall that the exponential distribution with a parameter $\delta>0$ is defined by $S_{X}(x)=\exp \{-\delta x\}, x \geq 0$. It is characterized by a functional equation

$$
S_{X}(x+t)=S_{X}(x) S_{X}(t) \quad \text { for all } x \geq 0 \text { independent of } t>0,
$$

postulating the classical univariate memory-less property. An immediate bivariate extension of the former relation is the functional equation

$$
S_{X_{1}, X_{2}}\left(x_{1}+t_{1}, x_{2}+t_{2}\right)=S_{X_{1}, X_{2}}\left(x_{1}, x_{2}\right) S_{X_{1}, X_{2}}\left(t_{1}, t_{2}\right)
$$

in the set $\mathcal{M}=\left\{x_{1}, x_{2}, t_{1}, t_{2} \geq 0\right\}$, representing the stronger version of bivariate lack of memory property. As a consequence of Theorem 1 in Aczél (1966), see pp. 215-216, its general solution is given by

$$
S_{X_{1}, X_{2}}\left(x_{1}, x_{2}\right)=\exp \left\{-\delta_{1} x_{1}-\delta_{2} x_{2}\right\} \quad \text { for some } \delta_{1}, \delta_{2}>0 \text {. }
$$

Marshall and Olkin (1967) did an alternative proof of the same statement and conclude in their Lemma 2.1 that the solution (3.2) of (3.1) leads to independent exponential marginal distributions with parameters $\delta_{1}$ and $\delta_{2}$.

Remark 3.1 (Two wrong counterexamples). The BLMP specified by (1.3) is a particular case of (3.1) when $t_{1}=t_{2}=t$ and the solution (3.2) is the independent solution of (1.3) when $\gamma_{3}=0$ in (1.2). Below we present our "counterexamples".

1. One can verify that the modified MO bivariate exponential distribution (1.4) being a solution of (1.5) is also solution of functional equation (3.1) in the special case when $t_{1}=\omega t$ and $t_{2}=t$. Hence, in addition to the independent solution (3.2) of (3.1) we got non-independent exponential random variables with parameters $\lambda_{1}+\lambda_{3}$ and $\lambda_{2}+\omega \lambda_{3}$ whose joint distribution (1.4) satisfies (3.1) as well. So, the first lucky impression is that we got a counterexample. Formally, this is true (as in BLMP case, that is, the joint survival function given by (1.2) is a solution of (3.1) when $\left.t_{1}=t_{2}=t\right)$. However, the reason of this wrong impression and "counterexample" is that the domain $\left\{x_{1}, x_{2}, t \geq 0\right.$ and $\left.\omega>0\right\}$ of functional equation (1.5) belongs to the domain $\mathcal{M}$ of (3.1). Therefore, not all solutions of (1.5) are solutions of (3.1). But, the independent solution (3.2) of functional equation (3.1) may serve as a particular solution of (1.5) and we will use this fact in the proof of Theorem 3.1. A nice related discussion can be found in Marshall and Olkin (1991). 
2. Interestingly, the MO bivariate exponential distribution (1.2) is the only solution with exponential marginals of functional equation (3.1) for all nonnegative $x_{1}, x_{2}, t_{1}, t_{2}$, but belonging to the set

$$
\left\{x_{1} \leq x_{2}, t_{1} \leq t_{2}\right\} \cup\left\{x_{1} \geq x_{2}, t_{1} \geq t_{2}\right\},
$$

being a subset of $\mathcal{M}$, see Marshall and Shaked (1979).

We will obtain a general solution of functional equation (1.5) in the next statement, or equivalently, we will characterize the W-BLMP.

\section{Theorem 3.1.}

(i) The non-negative continuous random vector $\left(X_{1}, X_{2}\right)$ possesses the $W$-BLMP defined by (1.5) if and only if its joint survival function is given by

$$
S_{X_{1}, X_{2}}\left(x_{1}, x_{2}\right)= \begin{cases}\exp \left\{-\theta x_{2}\right\} S_{X_{1}}\left(x_{1}-\omega x_{2}\right), & \text { if } x_{1} \geq \omega x_{2}, \\ \exp \left\{-\frac{\theta x_{1}}{\omega}\right\} S_{X_{2}}\left(x_{2}-\frac{x_{1}}{\omega}\right), & \text { if } x_{1} \leq \omega x_{2},\end{cases}
$$

for all $x_{1}, x_{2} \geq 0, \omega>0$ and some $\theta>0$, where $S_{X_{i}}\left(x_{i}\right)$ is the marginal survival function of $X_{i}, i=1,2$.

(ii) The only solution of (1.5) with exponential marginals is the modified MO bivariate exponential distribution (1.4).

Proof. Let $\left(X_{1}, X_{2}\right)$ possess W-BLMP, or equivalently, let (1.5) be true.

Set $x_{2}=0$ in (1.5) to get $S_{X_{1}, X_{2}}\left(x_{1}+\omega t, t\right)=S_{X_{1}}\left(x_{1}\right) S_{X_{1}, X_{2}}(\omega t, t)$. Substitute $x_{1}=x_{1}+\omega t$ and $x_{2}=t$ to conclude that

$$
S_{X_{1}, X_{2}}\left(x_{1}, x_{2}\right)=S_{X_{1}}\left(x_{1}-\omega x_{2}\right) S_{X_{1}, X_{2}}\left(\omega x_{2}, x_{2}\right), \quad \text { if } x_{1}-\omega x_{2} \geq 0 .
$$

As we noted in Remark 2.2, since the domain $\left\{x_{1}, x_{2}, t \geq 0\right.$ and $\left.\omega>0\right\}$ of (1.5) belongs to the domain $\mathcal{M}=\left\{x_{1}, x_{2}, t_{1}, t_{2} \geq 0\right\}$ of functional equation (3.1), it follows that the solution (3.2) of (3.1) may serve as a particular solution of (1.5), that is, it holds

$$
S_{X_{1}, X_{2}}\left(\omega x_{2}, x_{2}\right)=\exp \left\{-\left(\alpha_{1} \omega+\alpha_{2}\right) x_{2}\right\} \quad \text { for some } \alpha_{1}, \alpha_{2}>0 .
$$

Therefore, from (3.4) we obtain

$$
S_{X_{1}, X_{2}}\left(x_{1}, x_{2}\right)=\exp \left\{-\left(\alpha_{1} \omega+\alpha_{2}\right) x_{2}\right\} S_{X_{1}}\left(x_{1}-\omega x_{2}\right) \quad \text { if } x_{1}-\omega x_{2} \geq 0,
$$

which is the first relation in (3.3) letting $\theta=\alpha_{1} \omega+\alpha_{2}$.

By analogy, put $x_{1}=0$ in (1.5) to conclude that

$$
S_{X_{1}, X_{2}}\left(x_{1}, \frac{x_{1}}{\omega}\right)=\exp \left\{-\frac{\left(\beta_{1} \omega+\beta_{2}\right) x_{1}}{\omega}\right\}
$$

and to obtain the second expression in (3.3) with $\theta=\beta_{1} \omega+\beta_{2}$ for some $\beta_{1}>0$ and $\beta_{1}>0$ satisfying (3.2). 
In order to ensure the same joint survival function $S_{X_{1}, X_{2}}\left(x_{1}, x_{2}\right)$ along the line $L_{\omega}:\left\{x_{1}=\omega x_{2}\right\}$ one should require equality $\beta_{1} \omega+\beta_{2}=\alpha_{1} \omega+\alpha_{2}$ for all possible values of parameters involved. Therefore, (3.3) is valid.

Conversely, it is direct to check that (3.3) satisfies the functional equation (1.5) and the part (i) of the statement is established.

To prove (ii), suppose that $S_{X_{1}, X_{2}}\left(x_{1}, x_{2}\right)$ is a survival function solving functional equation (1.5). Then Theorem 3.1(i) implies its representation by (3.3). Assume that marginals of $\left(X_{1}, X_{2}\right)$ are exponentially distributed. This means that $S_{X_{1}}\left(x_{1}\right)=\exp \left\{-\alpha_{1} x_{1}\right\}$ and $S_{X_{2}}\left(x_{2}\right)=\exp \left\{-\beta_{2} x_{2}\right\}$ for all $x_{1}, x_{2} \geq 0$ and some $\alpha_{1}, \beta_{2}>0$. Replace these expressions in (3.3) to obtain

$$
S_{X_{1}, X_{2}}\left(x_{1}, x_{2}\right)= \begin{cases}\exp \left\{-\alpha_{1} x_{1}-\left(\theta-\omega \alpha_{1}\right) x_{2}\right\}, & \text { if } x_{1} \geq \omega x_{2}, \\ \exp \left\{-\frac{\left(\theta-\beta_{2}\right) x_{1}}{\omega}-\beta_{2} x_{2}\right\}, & \text { if } x_{1} \leq \omega x_{2} .\end{cases}
$$

In (3.5) substitute

$$
\omega \lambda_{1}=\theta-\beta_{2}, \quad \lambda_{2}=\theta-\omega \alpha_{1} \quad \text { and } \quad \omega \lambda_{3}=\omega \alpha_{1}+\beta_{2}-\theta .
$$

Note that $S_{X_{1}, X_{2}}\left(x_{1}, x_{2}\right)$ given by (3.5) is a survival function being non increasing in its arguments. Therefore, inequalities $\theta-\beta_{2} \geq 0$ and $\theta-\omega \alpha_{1} \geq 0$ have to be satisfied, i.e., $\lambda_{1}, \lambda_{2} \geq 0$ for $\omega>0$ and hence, $\omega \alpha_{1}+\beta_{2} \leq 2 \theta$.

We will show that the parameter $\lambda_{3}$ is non-negative as well. Following the brilliant method used by Gupta, Zeung and $\mathrm{Hu}$ (2010) in the proof of their Theorem 6.2, pp. 123-124, let $F_{X_{1}, X_{2}}\left(x_{1}, x_{2}\right)$ be the joint distribution of $\left(X_{1}, X_{2}\right)$. Then the function

$$
F\left(x_{1}\right)=F_{X_{1}, X_{2}}\left(\omega x_{1}, x_{1}\right)=1-S_{X_{1}}\left(\omega x_{1}\right)-S_{X_{2}}\left(x_{1}\right)+S_{X_{1}, X_{2}}\left(\omega x_{1}, x_{1}\right)
$$

defines a proper continuous univariate distribution function with a density

$$
f\left(x_{1}\right)=\frac{d}{d x_{1}} F\left(x_{1}\right)=\omega \alpha_{1} \exp \left\{-\omega \alpha_{1} x_{1}\right\}+\beta_{2} \exp \left\{-\beta_{2} x_{1}\right\}-\theta \exp \left\{-\theta x_{1}\right\} \geq 0 .
$$

Therefore, $\lim _{x_{1} \rightarrow 0^{+}} f\left(x_{1}\right)=\omega \alpha_{1}+\beta_{2}-\theta \geq 0$, i.e. $\omega \alpha_{1}+\beta_{2} \geq \theta$.

Thus, the parameter space $\left\{\alpha_{1}, \beta_{2}, \theta, \omega>0\right\}$ of $S_{X_{1}, X_{2}}\left(x_{1}, x_{2}\right)$ specified by (3.5) is restricted by the inequalities $\theta \leq \omega \alpha_{1}+\beta_{2} \leq 2 \theta$.

Finally, solving the system (3.6) we obtain

$$
\alpha_{1}=\lambda_{1}+\lambda_{3}, \quad \beta_{2}=\lambda_{2}+\omega \lambda_{3} \quad \text { and } \theta=\lambda_{1}+\lambda_{2}+\omega \lambda_{3}
$$

and restore (1.4) via (3.5). Note that at least one of parameters $\lambda_{i}, i=1,2,3$, should be positive, otherwise one would have a degenerate distribution. To finish the proof, it is easy to verify that (1.4) satisfies (1.5) indeed (as we did just before Remark 2.1).

Second proof of Theorem 3.1(i). Instead of using a particular solution (3.2) of functional equation (1.5) in the proof of Theorem 3.1, one can proceed as follows. 
Let (1.5) be true and set $x_{2}=0$ to get relation (3.4) when $x_{1} \geq \omega x_{2}$. Put $x_{1}=\omega t$ and $x_{2}=t$ in (3.4) to obtain the functional equation $S_{X_{1}, X_{2}}(2 \omega t, 2 t)=$ $\left[S_{X_{1}, X_{2}}(\omega t, t)\right]^{2}$ for all $t \geq 0$ and $\omega>0$. Iterating the procedure one will arrive to equation

$$
S_{X_{1}, X_{2}}(k \omega t, k t)=\left[S_{X_{1}, X_{2}}(\omega t, t)\right]^{k},
$$

which is valid for all $t \geq 0$ and $k=1,2, \ldots$ Let $\Gamma(t)=S_{X_{1}, X_{2}}(k \omega t, k t)$ and the last equality becomes

$$
\Gamma(t)=\left[\Gamma\left(\frac{t}{k}\right)\right]^{k} \quad \text { for all } t>0 \text { and } k=1,2, \ldots
$$

This relation is a consequence of the functional equation $\Gamma(x+t)=\Gamma(x) \Gamma(t)$ for $x, t \geq 0$, characterizing the exponential distribution. Therefore, $\Gamma(t)=\exp \{-\theta t\}$ for some $\theta>0$, see Section 5.3 in Galambos and Kotz (1978). Thus, we got the first expression in (3.3).

Using similar arguments one will obtain the second equation in (3.3) if $x_{1} \leq \omega x_{2}$.

Remark 3.2 (Independent case). In the proof of Theorem 3.1, we used the condition $\beta_{1} \omega+\beta_{2}=\alpha_{1} \omega+\alpha_{2}$ to ensure the same expression for $S_{X_{1}, X_{2}}\left(x_{1}, x_{2}\right)$ along the line $L_{\omega}:\left\{x_{1}=\omega x_{2}\right\}$. If it happens that $\alpha_{1}=\beta_{1}$ and $\alpha_{2}=\beta_{2}$, then the solution of the system (3.6) will indicate that $\lambda_{3}=0$, that is, $X_{1}$ and $X_{2}$ are independent and exponentially distributed. Only in this case the general solution (3.2) of functional equation (3.1) is independent solution of (1.5) as well, see an alternative proof of Corollary 4.2 .

\subsection{Multivariate weak lack of memory property}

Let us comment briefly the multivariate case. Denote by $S_{n}(\mathbf{x})$ the survival function of nonnegative random vector $\mathbf{X}=\left(X_{1}, \ldots, X_{n}\right)$, where $\mathbf{x}=\left(x_{1}, \ldots, x_{n}\right) \in$ $R_{+}^{n}, n \geq 2$.

The multivariate analog of functional equation (1.3) can be written as

$$
S_{n}((v+1) \mathbf{x})=S_{n}(v \mathbf{x}) S_{n}(\mathbf{x}) \quad \text { for all } v>0, \mathbf{x} \in R_{+}^{n} .
$$

It turns out that former equation can be equivalently represented by

$$
\left[S_{n}\left(\frac{t \mathbf{x}}{m}\right)\right]^{m}=S_{n}(t \mathbf{x}) \quad \text { for all } t>0, \mathbf{x} \in R_{+}^{n} \text { and } m=1,2, \ldots,
$$

see Theorem 3.1.b in Marshall and Olkin (1991). Therefore, the vector X has a distribution with exponential scaled minima, that is, $\min _{i \in I}\left\{v_{i} X_{i}\right\}$ has an exponential distribution for all $v_{i}>0, i=1, \ldots, n$ and all non-empty sets $I \in\{1,2, \ldots, n\}$. 
We will finish this section with a definition and characterization of a multivariate weak lack of memory property corresponding to functional equation (1.5). It is specified by relations

$$
S_{n}\left(x_{1}+x_{i} \omega, x_{2}+x_{i}, \ldots, x_{n}+x_{i}\right)=S_{n}\left(x_{1}, x_{2}, \ldots, x_{n}\right) S_{n}\left(x_{i} \omega, x_{i}, \ldots, x_{i}\right)
$$

for $i=1,2, \ldots, n$.

Definition 3.1. A random vector $\mathbf{X}$ is said to have a weak multivariate lack of memory property (to be abbreviated W-MLMP) if equation (3.7) holds.

Let $R_{n}(\mathbf{x})=-\ln S_{n}(\mathbf{x})$ and denote $\mathbf{1}_{\omega}=\left(1,1, \ldots, \omega^{-1}, \ldots, 1\right)$, for example, all elements of the vector $\mathbf{1}_{\omega}$ are equal to 1 , instead of $i$ th which is $\omega^{-1}$, $i=1,2, \ldots, n$. The following characterization of W-MLMP is true.

Theorem 3.2. The vector $\mathbf{X}$ have $W$-MLMP if and only if there exist numbers $\theta>0$ and $\omega>0$ such that the following statements are equivalent for all $\mathbf{x}>\mathbf{0}$ and $t>0$ with $\mathbf{x}-\mathbf{1}_{\omega} t \geq 0$ :

(i) $S_{n}(\mathbf{x})=\exp \left(-\frac{\theta t}{\omega}\right) S_{n}\left(\mathbf{x}-\mathbf{1}_{\omega} t\right)$;

(ii) $R_{n}(\mathbf{x})=\frac{\theta t}{\omega}+R_{n}\left(\mathbf{x}-\mathbf{1}_{\omega} t\right)$;

(iii) $R_{n}\left(\mathbf{x}+x \mathbf{1}_{\omega}\right)=R_{n}(\mathbf{x})+R_{n}\left(x \mathbf{1}_{\omega}\right)$.

Proof. Suppose $\mathbf{X}$ have W-MLMP, i.e., equation (3.7) holds. Thus,

$S_{n}(\mathbf{x})=S_{n}\left(x_{1}-t+t, \ldots, x_{i-1}-t+t, x_{i}-\frac{t}{\omega}+\frac{t}{\omega}, x_{i+1}-t+t, \ldots, x_{n}-t+t\right)$.

Applying (3.7) we get $S_{n}(\mathbf{x})=S_{n}\left(\mathbf{x}-\mathbf{1}_{\omega} t\right) S_{n}\left(\mathbf{1}_{\omega} t\right)$.

Taking $x_{i}=t$ for all $i=1, \ldots, n$ in (3.7) and substituting $G(\omega, t)=$ $S_{n}(\omega t, t, \ldots, t)$ we obtain $G(\omega+1, t)=G(\omega, t) G(1, t)$ which can be reduced to multiplicative Cauchy functional equation with a solution $G(\omega, t)=\exp \{-c \omega t\}$ for arbitrary constant $c>0$, see Aczél (1966), p. 35. Thus, $S\left(\mathbf{1}_{\omega} t\right)=\exp \left(-\frac{\theta}{\omega} t\right)$ and hence the relation (i) in the Theorem 3.2 is fulfilled.

The other equivalent implications are result of standard transformations (logarithmic and exponential), or can be checked trivially.

Let us note that when $\omega=1$ one will obtain the corresponding characterization of the multivariate lack of memory property established by Kulkarni (2006), see her Theorem 1.

\section{Marginal restrictions}

Theorem 3.1 characterizes bivariate continuous distributions in $R_{+}^{2}$ possessing the W-BLMP with joint survival function $S_{X_{1}, X_{2}}\left(x_{1}, x_{2}\right)$ specified by relation (3.3). 
It happens that the set of possible marginal distributions of bivariate continuous distributions possessing W-BLMP is restricted. In other words, the corresponding joint survival function is valid, for example, $\frac{\partial^{2} S_{X_{1}, X_{2}}\left(x_{1}, x_{2}\right)}{\partial x_{1} \partial x_{2}} \geq 0$, only for certain marginal distributions of $X_{1}$ and $X_{2}$. Kulkarni (2006) did a similar conclusion about marginals of bivariate distributions possessing BLMP.

Here we will obtain the associated constraints in terms of marginal densities and hazard rates. As a result, we will get the admissible values of the parameter $\theta$ in the joint survival function given by (3.3). Thus, one would be able to generate a wealth of bivariate distributions possessing W-BLMP having given marginals. We illustrate the methodology by several examples and establish related closure properties.

\subsection{Marginal density restrictions}

One can deduce that $S_{X_{1}, X_{2}}\left(x_{1}, x_{2}\right)$ specified by (3.3) admits the Lebesgue decomposition

$$
S_{X_{1}, X_{2}}\left(x_{1}, x_{2}\right)=(1-\alpha) S_{X_{1}, X_{2}}^{\mathrm{ac}}\left(x_{1}, x_{2}\right)+\alpha S_{X_{1}, X_{2}}^{\mathrm{si}}\left(x_{1}, x_{2}\right) \mathbf{I}_{\left\{x_{1}=\omega x_{2}\right\}},
$$

where $\alpha=P\left(X_{1}=\omega X_{2}\right) \geq 0, \mathbf{I}_{\{E\}}$ is the indicator function of the set $E$, $S_{X_{1}, X_{2}}^{\mathrm{si}}\left(x_{1}, x_{2}\right) \mathbf{I}_{\left\{x_{1}=\omega x_{2}\right\}}$ is a singular component with support along the line $L_{\omega}=$ $\left\{x_{1}=\omega x_{2}\right\}$ and $S_{X_{1}, X_{2}}^{\text {ac }}\left(x_{1}, x_{2}\right)$ is an absolutely continuous survival function. Hence, the joint continuous density $f_{X_{1}, X_{2}}\left(x_{1}, x_{2}\right)$ corresponding to (4.1) can be written as

$$
f_{X_{1}, X_{2}}\left(x_{1}, x_{2}\right)=(1-\alpha) f_{X_{1}, X_{2}}^{\mathrm{ac}}\left(x_{1}, x_{2}\right)+\alpha f_{X_{1}, X_{2}}^{\mathrm{si}}\left(x_{1}, x_{2}\right) \mathbf{I}_{\left\{x_{1}=\omega x_{2}\right\}} .
$$

Clearly, if $\alpha=P\left(X_{1}=\omega X_{2}\right)=0$, then the joint distribution of the random vector $\left(X_{1}, X_{2}\right)$ in (3.3) will be absolutely continuous.

The next theorem shows a list of associated constraints in terms of marginal densities.

Theorem 4.1. Let $X_{i}$ be a non-negative random variable with absolutely continuous density $f_{X_{i}}\left(x_{i}\right)$ on all finite intervals, $i=1,2$. Then $S_{X_{1}, X_{2}}\left(x_{1}, x_{2}\right)$ specified by (3.3) is a proper bivariate survival function if only if

$$
\theta \leq \omega f_{X_{1}}(0)+f_{X_{2}}(0) \leq 2 \theta
$$

and the following two inequalities are fulfilled

$$
\begin{array}{cc}
\theta+\omega \frac{d}{d x_{1}} \ln \left[f_{X_{1}}\left(x_{1}-\omega x_{2}\right)\right] \geq 0, & \text { for } x_{1} \geq \omega x_{2}, \\
\theta+\frac{d}{d x_{2}} \ln \left[f_{X_{2}}\left(x_{2}-\frac{x_{1}}{\omega}\right)\right] \geq 0, & \text { for } x_{1} \leq \omega x_{2} .
\end{array}
$$


Proof. Since the survival function $S_{X_{1}, X_{2}}\left(x_{1}, x_{2}\right)$ may have a singular component along the line $L_{\omega}=\left\{x_{1}=\omega x_{2}\right\}$, then $S_{X_{1}, X_{2}}\left(x_{1}, x_{2}\right)$ given by (3.3) will be proper if and only if both the absolutely continuous part $S_{X_{1}, X_{2}}^{\mathrm{ac}}\left(x_{1}, x_{2}\right)$ and the singular part $S_{X_{1}, X_{2}}^{\text {si }}\left(x_{1}, x_{2}\right) \mathbf{I}_{\left\{x_{1}=\omega x_{2}\right\}}$ are survival functions and satisfy (4.1) with a singularity weight $\alpha=P\left(X_{1}=\omega X_{2}\right)$.

Starting from (3.3), after some algebra one will find that the function $(1-\alpha) f_{X_{1}, X_{2}}^{\text {ac }}\left(x_{1}, x_{2}\right)$ can be written as

$$
\left\{\begin{array}{l}
\theta \exp \left(-\theta x_{2}\right) f_{X_{1}}\left(x_{1}-\omega x_{2}\right)+\omega \exp \left(-\theta x_{2}\right) \frac{d}{d x_{1}} f_{X_{1}}\left(x_{1}-\omega x_{2}\right) \\
\quad \text { if } x_{1} \geq \omega x_{2} \\
\frac{\theta}{\omega} \exp \left(-\frac{\theta x_{1}}{\omega}\right) f_{X_{2}}\left(x_{2}-\frac{x_{1}}{\omega}\right)+\frac{1}{\omega} \exp \left(-\frac{\theta x_{1}}{\omega}\right) \frac{d}{d x_{2}} f_{X_{2}}\left(x_{2}-\frac{x_{1}}{\omega}\right) \\
\quad \text { if } x_{1} \leq \omega x_{2} .
\end{array}\right.
$$

By assumption, densities $f_{X_{i}}\left(x_{i}\right)$ are absolutely continuous on all finite intervals, $i=1,2$. This implies that the derivative $\frac{d}{d x_{i}} f_{X_{i}}\left(x_{i}\right)$ is integrable on $(-\infty, \infty)$, see Block and Basu (1974) for related discussion. So, we can compute

$$
P\left(X_{1}>\omega X_{2}\right)=\int_{0}^{\infty} \int_{0}^{\frac{x_{1}}{\omega}}(1-\alpha) f_{X_{1}, X_{2}}^{\mathrm{ac}}\left(x_{1}, x_{2}\right) d x_{2} d x_{1}=1-\frac{\omega f_{X_{1}}(0)}{\theta}
$$

and

$$
P\left(X_{1}<\omega X_{2}\right)=\int_{0}^{\infty} \int_{0}^{\omega x_{2}}(1-\alpha) f_{X_{1}, X_{2}}^{\mathrm{ac}}\left(x_{1}, x_{2}\right) d x_{1} d x_{2}=1-\frac{f_{X_{2}}(0)}{\theta} .
$$

But $\alpha=P\left(X_{1}=\omega X_{2}\right)=1-P\left(X_{1}>\omega X_{2}\right)-P\left(X_{1}<\omega X_{2}\right)$ and we conclude that

$$
\alpha=-1+\frac{1}{\theta}\left[\omega f_{X_{1}}(0)+f_{X_{2}}(0)\right] \in[0,1]
$$

which implies inequalities (4.2).

$$
\begin{aligned}
& \text { Now, since } \frac{\partial^{2} S_{X_{1}, X_{2}}\left(x_{1}, x_{2}\right)}{\partial x_{1} \partial x_{2}} \text { should be non-negative, from (4.4) we obtain } \\
& \exp \left(-\theta x_{2}\right)\left[\theta f_{X_{1}}\left(x_{1}-\omega x_{2}\right)+\omega \frac{d}{d x_{1}} f_{X_{1}}\left(x_{1}-\omega x_{2}\right)\right] \geq 0, \quad \text { if } x_{1} \geq \omega x_{2}
\end{aligned}
$$

and

$$
\omega^{-1} \exp \left(-\frac{\theta x_{1}}{\omega}\right)\left[\theta f_{X_{2}}\left(x_{2}-\frac{x_{1}}{\omega}\right)+\frac{d}{d x_{2}} f_{X_{2}}\left(x_{2}-\frac{x_{1}}{\omega}\right)\right] \geq 0, \quad \text { if } x_{1} \leq \omega x_{2} .
$$

Taking into account that $f_{X_{1}}\left(x_{1}-\omega x_{2}\right) \geq 0$ for $x_{1} \geq \omega x_{2}$ and $f_{X_{2}}\left(x_{2}-\frac{x_{1}}{\omega}\right) \geq 0$ when $x_{1} \leq \omega x_{2}$, we arrive to inequalities (4.3a) and (4.3b). 
Remark 4.1 (Exponential case). In the proof of Theorem 3.1(ii), we established that $\theta \leq \omega \alpha_{1}+\beta_{2} \leq 2 \theta$, where $\alpha_{1}$ and $\beta_{2}$ are parameters of exponentially distributed random variables $X_{1}$ and $X_{2}$, correspondingly. The last inequalities are particular case of restrictions (4.2) since $f_{X_{1}}(0)=\alpha_{1}$ and $f_{X_{2}}(0)=\beta_{2}$. In this case, (4.3a) and (4.3b) are satisfied as well.

Following the corresponding procedure used in the proof of Theorem 5.1 in Marshall and Olkin (1967), we get the following lemma.

Lemma 4.1. The singular component of $S_{X_{1}, X_{2}}\left(x_{1}, x_{2}\right)$ specified by (3.3) is given by

$$
S_{X_{1}, X_{2}}^{\mathrm{si}}\left(x_{1}, x_{2}\right) \mathbf{I}_{\left\{x_{1}=\omega x_{2}\right\}}=\exp \left\{-\theta \max \left(x_{1}, \omega x_{2}\right)\right\} .
$$

If set $\omega=1$ in expression given by Lemma 4.1, one will get the singular component in BLMP case, see equation (5.6) in Marshall and Olkin (1967).

Notice that if inequalities (4.3a) and (4.3b) are fulfilled for some $\theta=\theta_{0}>0$, then they are also satisfied for all $\theta>\theta_{0}$. Denote by

$\tau=\{$ the greatest lower bound of $\theta$-values satisfying (4.3a) and (4.3b) $\}$.

Thus $\tau$ is a function of marginal parameters and $\omega$. The range of possible values of $\theta$ is given in Lemma 4.2.

Lemma 4.2. Suppose that $\theta \leq \omega f_{X_{1}}(0)+f_{X_{2}}(0)$. If

$$
\theta \in\left[\max \left\{\tau, \max \left(\omega f_{X_{1}}(0), f_{X_{2}}(0)\right)\right\}, \omega f_{X_{1}}(0)+f_{X_{2}}(0)\right]
$$

then Theorem 4.1 is verified. Moreover, the joint distribution of $\left(X_{1}, X_{2}\right)$ given by (3.3) is absolutely continuous if and only if $\theta=\omega f_{X_{1}}(0)+f_{X_{2}}(0)$.

Proof. We know from the proof of Theorem 4.1 that

$$
P\left(X_{1}>\omega X_{2}\right)=1-\frac{\omega f_{X_{1}}(0)}{\theta} \geq 0 \quad \text { and hence } \quad \omega f_{X_{1}}(0) \leq \theta .
$$

By analogy, from the expression for $P\left(X_{1}<\omega X_{2}\right)$ we obtain $f_{X_{2}}(0) \leq \theta$. Thus, $\theta \geq \max \left\{\omega f_{X_{1}}(0), f_{X_{2}}(0)\right\}$.

Depending on the parameters of marginal densities, it may happen that $\tau>$ $\omega f_{X_{1}}(0)+f_{X_{2}}(0)$ in some special cases, which would contradict inequalities (4.2). Such high $\tau$-values would be outside of the parameter space of W-BLMP. The lower bound in (4.5) can be obtained by checking the restrictions on $\theta$ imposed by inequalities (4.3a) and (4.3b) in order to get the associated value of $\tau$.

When $\alpha=P\left(X_{1}=\omega X_{2}\right)=0$, then $\omega f_{X_{1}}(0)+f_{X_{2}}(0)-\theta=0$. Since $\alpha \in[0,1]$ and $\omega f_{X_{1}}(0)+f_{X_{2}}(0)$ is increasing function on $\omega$ we always have $\theta \leq \omega f_{X_{1}}(0)+$ $f_{X_{2}}(0)$, which is the upper bound for $\theta$ in (4.5).

Finally, observe that the joint density function is absolutely continuous if and only if $\alpha=0$, i.e. when $\theta=\omega f_{X_{1}}(0)+f_{X_{2}}(0)$. This completes the proof. 
Denote by $\Theta=(\theta, \omega, \Lambda)$ the parameter vector of W-BLMP class of distributions, where $\Lambda$ is the vector of parameters of marginal distributions. The parameter space of W-BLMP is well defined when $\theta$ belongs to the closed interval given in (4.5). It is crucial for construction of proper bivariate continuous distributions possessing W-BLMP from pre-specified marginal densities as we will see in the next example.

Example 4.1. Let $c_{1}, c_{2}, c_{3}>0$ and the densities $f_{X_{1}}\left(x_{1}\right)$ and $f_{X_{2}}\left(x_{2}\right)$ be given by

$$
f_{X_{1}}\left(x_{1}\right)=\left(c_{1}-\frac{c_{3}}{c_{2}+x_{1}^{2}}\right) \exp \left\{-c_{1} x_{1}+\frac{c_{3}}{\sqrt{c_{2}}} \tan ^{-1}\left(\frac{x_{1}}{\sqrt{c_{2}}}\right)\right\}, \quad x_{1} \geq 0,
$$

and

$$
f_{X_{2}}\left(x_{2}\right)=\omega\left(c_{1}-\frac{c_{3}}{c_{2}+\omega^{2} x_{2}^{2}}\right) \exp \left\{-c_{1} \omega x_{2}+\frac{c_{3}}{\sqrt{c_{2}}} \tan ^{-1}\left(\frac{\omega}{\sqrt{c_{2}}} x_{2}\right)\right\}, \quad x_{2} \geq 0
$$

with $\omega>0$. In both cases the condition $c_{1} \geq \frac{c_{3}}{c_{2}}$ ensures that $f_{X_{i}}(0) \geq 0, i=1,2$.

On Figure 6 we show the form of density $f_{X_{2}}\left(x_{2}\right)$ for fixed $c_{1}, c_{2}, c_{3}$ and four different values $(0.78,1.0,1.2$ and 1.44$)$ of the parameter $\omega$. One can detect the impact of the parameter $\omega$ on the skewness and leptokurtosis of the density of $X_{2}$.

(a)

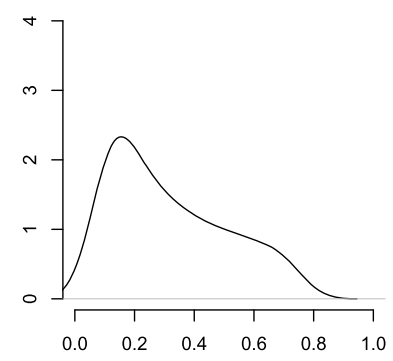

(c)

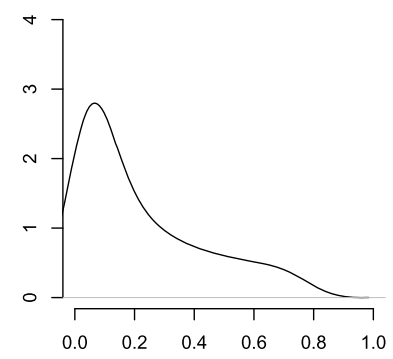

(b)

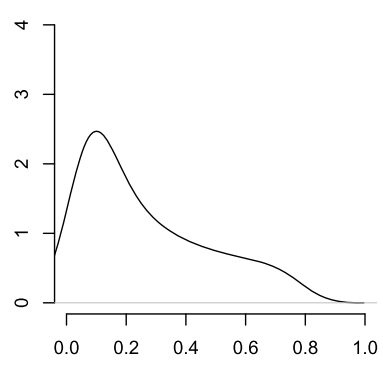

(d)

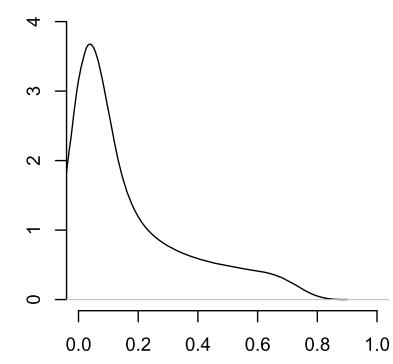

Figure 6 Marginal density $f_{X_{2}}\left(x_{2}\right)$ in Example 4.1 with $c_{1}=1, c_{2}=3$ and $c_{3}=1$ for (a) $\omega=0.78$, (b) $\omega=1.0$, (c) $\omega=1.2$ and (d) $\omega=1.44$. 
Consider a joint density $f_{X_{1}, X_{2}}\left(x_{1}, x_{2}\right)$ represented by

$$
\left\{\begin{aligned}
\left\{\frac{c_{3} \omega}{\left[c_{2}+\left(x_{1}-\omega x_{2}\right)^{2}\right]^{2}}+\frac{c_{3}\left(2 c_{1} \omega-\theta\right)}{c_{2}+\left(x_{1}-\omega x_{2}\right)^{2}}-c_{1}\left(c_{1} \omega-\theta\right)\right\} & \\
\quad \times \exp \left\{-\left[c_{1} x_{1}+\left(\theta-c_{1} \omega\right) x_{2}-\frac{c_{3}}{\sqrt{c_{2}}} \tan ^{-1}\left(\frac{x_{1}-\omega x_{2}}{\sqrt{c_{2}}}\right)\right]\right\}, & \text { if } x_{1} \geq \omega x_{2}, \\
\left\{\frac{c_{3} \omega\left[2\left(\omega x_{2}-x_{1}\right)-c_{3}\right]}{\left[c_{2}+\left(\omega x_{2}-x_{1}\right)^{2}\right]^{2}}+\frac{c_{3}\left(2 c_{1} \omega-\theta\right)}{c_{2}+\left(\omega x_{2}-x_{1}\right)^{2}}-c_{1}\left(c_{1} \omega-\theta\right)\right\} & \\
\quad \times \exp \left\{-\left[c_{1} \omega x_{2}+\left(\frac{\theta}{\omega}-c_{1}\right) x_{1}-\frac{c_{3}}{\sqrt{c_{2}}} \tan ^{-1}\left(\frac{\omega x_{2}-x_{1}}{\sqrt{c_{2}}}\right)\right]\right\}, & \text { if } x_{1} \leq \omega x_{2} .
\end{aligned}\right.
$$

One can verify that $f_{X_{1}, X_{2}}\left(x_{1}, x_{2}\right)$ has marginals $f_{X_{1}}\left(x_{1}\right)$ and $f_{X_{2}}\left(x_{2}\right)$ indeed. The parameter space in this case is $\Theta=(\theta, \omega, \Lambda)$ with $\Lambda=\left(c_{1}, c_{2}, c_{3}\right)$.

Applying (4.4) we obtain that $(1-\alpha) f_{X_{1}, X_{2}}^{\text {ac }}\left(x_{1}, x_{2}\right)$ is given by

$$
\left\{\begin{aligned}
\theta\left\{\frac{c_{3} \omega\left[2\left(x_{1}-\omega x_{2}\right)-c_{3}\right]}{\left[c_{2}+\left(x_{1}-\omega x_{2}\right)^{2}\right]^{2}}+\frac{c_{3}\left(2 c_{1} \omega-1\right)}{c_{2}+\left(x_{1}-\omega x_{2}\right)^{2}}-c_{1}\left(c_{1} \omega-1\right)\right\} & \\
\quad \times \exp \left\{-\left[c_{1} x_{1}+\left(\theta-c_{1} \omega\right) x_{2}-\frac{c_{3}}{\sqrt{c_{2}}} \tan ^{-1}\left(\frac{x_{1}-\omega x_{2}}{\sqrt{c_{2}}}\right)\right]\right\}, & \text { if } x_{1} \geq \omega x_{2}, \\
\theta\left\{\frac{c_{3} \omega\left[2\left(\omega x_{2}-x_{1}\right)-c_{3}\right]}{\left[c_{2}+\left(\omega x_{2}-x_{1}\right)^{2}\right]^{2}}+\frac{c_{3}\left(2 c_{1} \omega-1\right)}{c_{2}+\left(\omega x_{2}-x_{1}\right)^{2}}-c_{1}\left(c_{1} \omega-1\right)\right\} & \\
\quad \times \exp \left\{-\left[c_{1} \omega x_{2}+\left(\frac{\theta}{\omega}-c_{1}\right) x_{1}-\frac{c_{3}}{\sqrt{c_{2}}} \tan ^{-1}\left(\frac{\omega x_{2}-x_{1}}{\sqrt{c_{2}}}\right)\right]\right\}, & \text { if } x_{1} \leq \omega x_{2} .
\end{aligned}\right.
$$

After some algebra we obtain $\alpha=P\left(X_{1}=\omega X_{2}\right)=\frac{2 \omega}{\theta}\left(c_{1}-\frac{c_{3}}{c_{2}}\right)-1$.

If $x_{1}=\omega x_{2}=x \geq 0$, for the singular component of $S_{X_{1}, X_{2}}\left(x_{1}, x_{2}\right)$ from Lemma 4.1 we obtain

$S_{X_{1}, X_{2}}^{\mathrm{si}}\left(x, \frac{x}{\omega}\right)=\exp \left\{-\frac{\theta x}{\omega}\right\} \quad$ and therefore $\alpha f_{X_{1}, X_{2}}^{\mathrm{si}}\left(x, \frac{x}{\omega}\right)=\frac{\theta x}{\omega} \exp \left\{-\frac{\theta x}{\omega}\right\}$.

Figure 7 displays the shape of the density $f_{X_{1}, X_{2}}\left(x_{1}, x_{2}\right)$ for the same fixed values of parameters, for example, $c_{1}=1, c_{2}=3, c_{3}=1$ and $\omega$ used in the graphs of Figure 6.

The corresponding lines of singularity $L_{\omega}$ are colored in red. Note that the parameter $\omega$ introduces a certain asymmetry on the form of bivariate density especially in the upper tails. When $\omega=1$, we have $f_{X_{1}}\left(x_{1}\right)=f_{X_{2}}\left(x_{2}\right)$ for all $x_{1}, x_{2} \geq 0$. This particular case, see Figure 7(b), has been considered by Kulkarni (2006).

Restrictions (4.3a) and (4.3b) imply that if $x_{1} \geq \omega x_{2}$, then

$$
\theta+\frac{2 \omega c_{3}\left(x_{1}-\omega x_{2}\right)}{\left\{c_{1}\left[c_{2}+\left(x_{1}-\omega x_{2}\right)^{2}\right]-c_{3}\right\}\left[c_{2}+\left(x_{1}-\omega x_{2}\right)^{2}\right]}-\omega\left[c_{1}-\frac{c_{3}}{c_{2}+\left(x_{1}-\omega x_{2}\right)^{2}}\right] \geq 0
$$

and if $x_{1} \leq \omega x_{2}$ we get

$$
\theta+\frac{2 \omega c_{3}\left(\omega x_{2}-x_{1}\right)}{\left\{c_{1}\left[c_{2}+\left(\omega x_{2}-x_{1}\right)^{2}\right]-c_{3}\right\}\left[c_{2}+\left(\omega x_{2}-x_{1}\right)^{2}\right]}-\omega\left[c_{1}-\frac{c_{3}}{c_{2}+\left(\omega x_{2}-x_{1}\right)^{2}}\right] \geq 0
$$


(a)

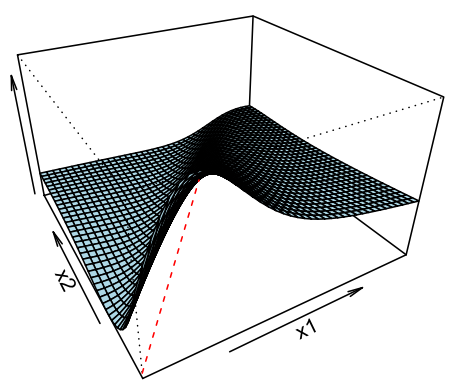

(c)

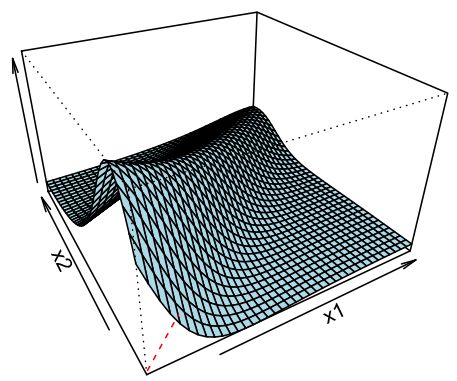

(b)

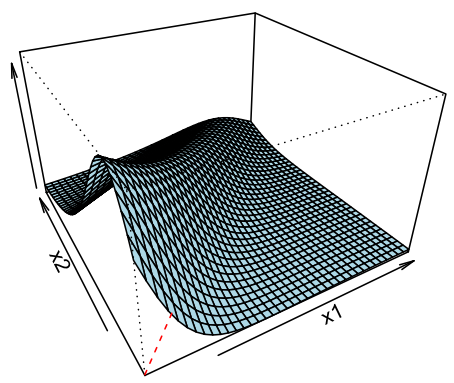

(d)

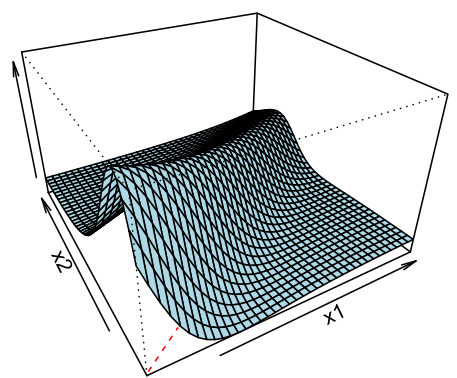

Figure 7 Bivariate density $f_{X_{1}, X_{2}}\left(x_{1}, x_{2}\right)$ with $c_{1}=1, c_{2}=3, c_{3}=1$ and $\theta=1$ for (a) $\omega=0.78$, (b) $\omega=1.0$, (c) $\omega=1.2$ and (d) $\omega=1.44$.

These functions are increasing in $x_{1}$ and $x_{2}$, with a minimum at the point $(0,0)$. Hence, from definition of $\tau$ we have $\tau=\omega\left(c_{1}-\frac{c_{3}}{c_{2}}\right)$ and using (4.5) for the possible values of $\theta$ we obtain the interval $\left[\omega\left(c_{1}-\frac{c_{3}}{c_{2}}\right), 2 \omega\left(c_{1}-\frac{c_{3}}{c_{2}}\right)\right]$. When $c_{1}=1, c_{2}=3$ and $c_{3}=1$, we obtain $\theta \in\left[\frac{2 \omega}{3}, \frac{4 \omega}{3}\right]$. The graphs on Figure 7 are plotted when $\theta=1$. We selected this value since it belongs to the interval $\left[\frac{2 \omega}{3}, \frac{4 \omega}{3}\right]$ for all chosen values $(0.78,1.0,1.2$ and 1.44$)$ of the parameter $\omega$, i.e., all bivariate distributions have a singular part.

If it happens that $\theta=2 \omega\left(c_{1}-\frac{c_{3}}{c_{2}}\right)$, the corresponding bivariate distribution will be absolutely continuous according to Lemma 4.1. When $c_{1}=1, c_{2}=3, c_{3}=1$, we obtain $\theta=\frac{4 \omega}{3}$ and Figure 8 shows the shape of absolutely continuous densities corresponding to cases (a)-(d) displayed on Figure 7.

\subsection{Marginal failure rate restrictions}

Denote by $r_{X_{i}}\left(x_{i}\right)$ the marginal failure rate of a continuous random variable $X_{i}$, that is, $r_{X_{i}}\left(x_{i}\right)=\frac{f_{X_{i}}\left(x_{i}\right)}{S_{X_{i}}\left(x_{i}\right)}, x_{i} \geq 0$ for $i=1,2$. The last equality indicates that the 
(a)

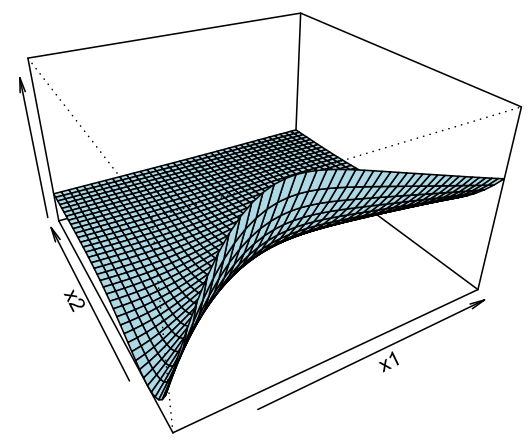

(c)

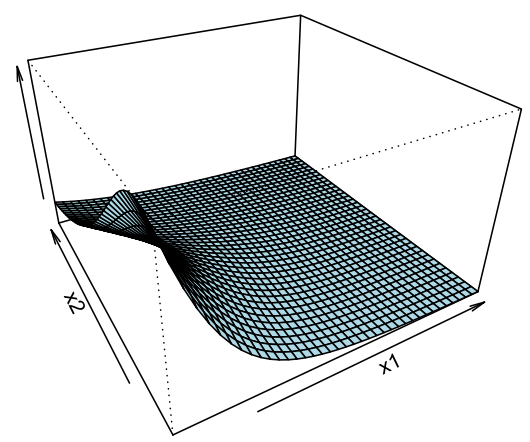

(b)

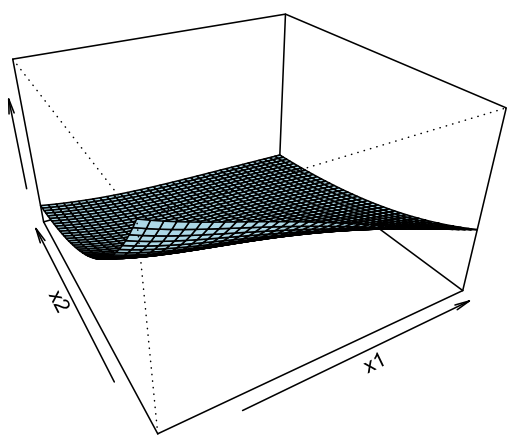

(d)

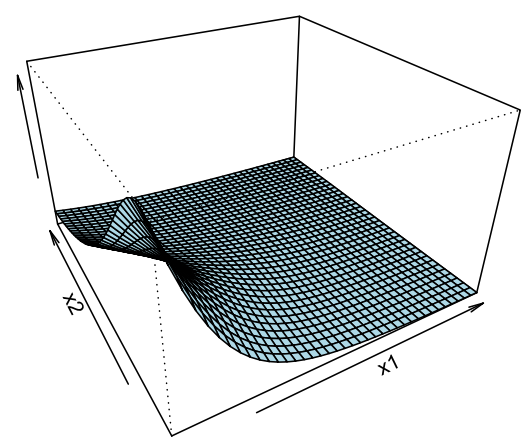

Figure 8 Absolutely continuous $f_{X_{1}, X_{2}}\left(x_{1}, x_{2}\right)$ for $c_{1}=1, c_{2}=3, c_{3}=1$. (a) $\omega=0.78$ and $\theta=1.04$; (b) $\omega=1.0$ and $\theta=1.3334$; (c) $\omega=1.2$ and $\theta=1.6$ and (d) $\omega=1.44$ with $\theta=1.92$.

relations from Section 4.1 treating marginal densities can be rewritten in terms of marginal failure rates.

First, note that the condition

$$
\int_{0}^{\infty} r_{X_{i}}(x) d x=\infty, \quad i=1,2
$$

is necessary for $r_{X_{i}}(x)$ to be a failure rate, see Barlow and Proschan (1981).

On the other hand, the conditional failure rates $r_{i}\left(x_{1}, x_{2}\right)$ introduced in Johnson and Kotz (1975) by $r_{i}\left(x_{1}, x_{2}\right)=\frac{\partial}{\partial x_{i}}\left[-\ln S_{X_{1}, X_{2}}\left(x_{1}, x_{2}\right)\right]$ should be non-negative, $i=1,2$. Remind that the hazard vector $\left(r_{1}\left(x_{1}, x_{2}\right), r_{2}\left(x_{1}, x_{2}\right)\right)$ uniquely determines the joint distribution of $\left(X_{1}, X_{2}\right)$ in terms of line integral, see Marshall (1975).

Using (3.3) and conditions $r_{i}\left(x_{1}, x_{2}\right) \geq 0, i=1,2$, we obtain following inequalities

$$
0 \leq r_{X_{1}}\left(x_{1}\right) \leq \frac{\theta}{\omega} \quad \text { and } \quad 0 \leq r_{X_{2}}\left(x_{2}\right) \leq \theta \quad \text { for all } x_{1}, x_{2} \geq 0 .
$$


Limitations (4.6) and (4.7) indicate that the marginal failure rates $r_{X_{1}}\left(x_{1}\right)$ and $r_{X_{2}}\left(x_{2}\right)$ of W-BLMP should

- have unbounded support;

- be restricted from above by the horizontal lines $l_{1}: x_{1}=\frac{\theta}{\omega}$ and $l_{2}: x_{2}=\theta$, correspondingly.

Additional constraints on marginal failure rates are given in the following statement, being a consequence of Theorem 4.1 .

Corollary 4.1. Let $X_{i}$ be a non-negative random variable with differentiable failure rates $r_{X_{i}}\left(x_{i}\right)$ on all finite intervals, $i=1,2$. Then $S_{X_{1}, X_{2}}\left(x_{1}, x_{2}\right)$ specified by (3.3) is a proper bivariate survival function if only if

$$
\theta \leq \omega r_{X_{1}}(0)+r_{X_{2}}(0) \leq 2 \theta
$$

and

$$
\begin{aligned}
\theta-\omega r_{X_{1}}\left(x_{1}-\omega x_{2}\right)+\omega \frac{d}{d x_{1}} \ln \left[r_{X_{1}}\left(x_{1}-\omega x_{2}\right)\right] \geq 0, \quad \text { for } x_{1} \geq \omega x_{2}, \\
\theta-r_{X_{2}}\left(x_{2}-\frac{x_{1}}{\omega}\right)+\frac{d}{d x_{2}} \ln \left[r_{X_{2}}\left(x_{2}-\frac{x_{1}}{\omega}\right)\right] \geq 0, \quad \text { for } x_{1} \leq \omega x_{2} .
\end{aligned}
$$

Proof. Really, limitations (4.8) follow from (4.2), since $r_{X_{i}}(0)=f_{X_{i}}(0)$ for $i=1,2$.

The condition (4.9a) can be obtained from (4.3a), by using the relation

$\frac{d}{d x_{1}}\left[\ln f_{X_{1}}\left(x_{1}-\omega x_{2}\right)\right]=\frac{d}{d x_{1}}\left[\ln r_{X_{1}}\left(x_{1}-\omega x_{2}\right)\right]-r_{X_{1}}\left(x_{1}-\omega x_{2}\right) \quad$ for $x_{1} \geq \omega x_{2}$.

Similarly, (4.9b) is a consequence of (4.3b) when $x_{1} \leq \omega x_{2}$.

In terms of failure rates, the interval (4.5) of admissible values of $\theta$ transforms into

$$
\theta \in\left[\max \left\{\tau, \max \left(\omega r_{X_{1}}(0), r_{X_{2}}(0)\right)\right\}, \omega r_{X_{1}}(0)+r_{X_{2}}(0)\right]
$$

where $\tau$ is the greatest lower bound of $\theta$-values satisfying (4.9a) and (4.9b).

Notice that if the marginal failure rates are increasing functions, then $\tau=$ $\max \left(\omega r_{X_{1}}(0), r_{X_{2}}(0)\right)$ and therefore relation (4.10) can be written as

$$
\theta \in\left[\max \left(\omega r_{X_{1}}(0), r_{X_{2}}(0)\right), \omega r_{X_{1}}(0)+r_{X_{2}}(0)\right] .
$$

We link above conditions in Theorem 4.2 which enable to generate bivariate distributions possessing W-BLMP from given (pre-specified) marginal failure rates. 
Theorem 4.2. Let the marginal failure rates $r_{X_{i}}\left(x_{i}\right)$ be differentiable functions. If relations (4.6) to (4.10) hold, then the function $S_{X_{1}, X_{2}}\left(x_{1}, x_{2}\right)$ given by (3.3) is a proper joint survival function having $W-B L M P$ with marginals

$$
S_{X_{i}}(x)=\exp \left(-\int_{0}^{x} r_{X_{i}}(t) d t\right), \quad x \geq 0, i=1,2 .
$$

Theorem 4.2 is a counterpart of a similar statement treating BLMP case given by Kulkarni (2006), see her Theorem 3. The converse of Theorem 4.2 also holds for non-degenerate distributions and the statement is given below.

Proposition 4.1. If $S_{X_{1}, X_{2}}\left(x_{1}, x_{2}\right)$ is non-degenerate bivariate survival function given by equation (3.3) and has differentiable marginal failure rates then it must satisfy conditions (4.6) to (4.10).

Proof. Follows step by step the proof of Proposition 1 in Kulkarni (2006).

4.2.1 Examples. Here we generate bivariate distributions possessing W-BLMP from their given (pre-specified) marginal failure rates $r_{X_{1}}\left(x_{1}\right)$ and $r_{X_{2}}\left(x_{2}\right)$ that are increasing or/and decreasing functions. After checking restrictions (4.6) to (4.10), we use (4.12) to get the marginal survival functions $S_{X_{i}}\left(x_{i}\right), i=1,2$, and then apply (3.3) to obtain the corresponding valid joint survival function $S_{X_{1}, X_{2}}\left(x_{1}, x_{2}\right)$.

Example 4.2 (Example 4.1 continued, with increasing marginal failure rates). If we adopt the marginal densities $f_{X_{1}}\left(x_{1}\right)$ and $f_{X_{2}}\left(x_{2}\right)$ from Example 4.1, we will obtain the corresponding marginal failure rates given by $r_{X_{1}}\left(x_{1}\right)=c_{1}-\frac{c_{3}}{c_{2}+x_{1}^{2}}$ for $x_{1} \geq 0$ and $r_{X_{2}}\left(x_{2}\right)=\omega\left(c_{1}-\frac{c_{3}}{c_{2}+\omega^{2} x_{2}^{2}}\right)$ for $x_{2} \geq 0$ where $c_{2}, c_{3}, \omega>0$ and $c_{1} \geq \frac{c_{3}}{c_{2}}$. In this case, $r_{X_{1}}\left(x_{1}\right)$ and $r_{X_{2}}\left(x_{2}\right)$ are increasing functions of their arguments and we will obtain the interval of admissible values of $\theta$ from (4.11). Since $r_{X_{1}}(0)=$ $c_{1}-\frac{c_{3}}{c_{2}}$ and $r_{X_{2}}(0)=\omega\left(c_{1}-\frac{c_{3}}{c_{2}}\right)$ we have

$$
\theta \in\left[\omega\left(c_{1}-\frac{c_{3}}{c_{2}}\right), 2 \omega\left(c_{1}-\frac{c_{3}}{c_{2}}\right)\right] .
$$

Naturally, we got the same interval for possible values of the parameter $\theta$ as earlier. Using (3.3) we find the corresponding valid joint survival function

$$
S\left(x_{1}, x_{2}\right)=\left\{\begin{array}{l}
\exp \left\{-\left[c_{1} x_{1}+\left(\theta-c_{1} \omega\right) x_{2}-\frac{c_{3}}{\sqrt{c_{2}}} \tan ^{-1}\left(\frac{x_{1}-\omega x_{2}}{\sqrt{c_{2}}}\right)\right]\right\}, \\
\quad \text { if } x_{1} \geq \omega x_{2}, \\
\exp \left\{-\left[c_{1} \omega x_{2}+\left(\frac{\theta}{\omega}-c_{1}\right) x_{1}-\frac{c_{3}}{\sqrt{c_{2}}} \tan ^{-1}\left(\frac{\omega}{\sqrt{c_{2}}}\left(x_{2}-\frac{x_{1}}{\omega}\right)\right)\right]\right\}, \\
\quad \text { if } x_{1} \leq \omega x_{2} .
\end{array}\right.
$$


Example 4.3 (increasing marginal failure rates). For $x_{1}, x_{2} \geq 0, c_{1}, \omega>0$ and $0 \leq c_{2} \leq 1$, define $r_{X_{1}}\left(x_{1}\right)=\frac{\exp \left(c_{1} x_{1}\right)}{c_{2}+\exp \left(c_{1} x_{1}\right)}$ and $r_{X_{2}}\left(x_{2}\right)=\frac{\omega \exp \left(c_{1} x_{2}\right)}{c_{2}+\exp \left(\omega c_{1} x_{2}\right)}$. The marginal failure rates $r_{X_{1}}\left(x_{1}\right)$ and $r_{X_{2}}\left(x_{2}\right)$ are increasing functions with $r_{X_{1}}(0)=$ $\frac{\omega}{1+c_{2}}$ and $r_{X_{2}}(0)=\frac{2 \omega}{1+c_{2}}$. Thus, from (4.11) we conclude that $\theta \in\left[\frac{\omega}{1+c_{2}}, \frac{2 \omega}{1+c_{2}}\right]$. Applying (3.3), we obtain the proper joint survival function

$$
S\left(x_{1}, x_{2}\right)= \begin{cases}\exp \left\{-\left[\theta x_{2}+\frac{1}{c_{1}} \ln \frac{c_{2}+\exp \left(c_{1} x_{1}-c_{1} \omega x_{2}\right)}{c_{2}+1}\right]\right\}, & \text { if } x_{1} \geq \omega x_{2}, \\ \exp \left\{-\left[\frac{\theta x_{1}}{\omega}+\frac{1}{c_{1}} \ln \frac{c_{2}+\exp \left(c_{1} \omega x_{2}-c_{1} x_{1}\right)}{c_{2}+1}\right]\right\}, & \text { if } x_{1} \leq \omega x_{2},\end{cases}
$$

and it is absolutely continuous if $\theta=\frac{2 \omega}{1+c_{2}}$, according to Lemma 4.1.

Let us note that the admissible values of the marginal distribution parameters may be further limited as a consequence of inequalities (4.9a) and (4.9b), as illustrated in the next example.

Example 4.4 (Increasing and decreasing marginal failure rates). Let $r_{X_{1}}\left(x_{1}\right)=$ $\frac{1}{1+x_{1}}$ and $r_{X_{2}}\left(x_{2}\right)=\omega\left(2 \omega c_{2} x_{2}+c_{3}\right)$ for $x_{1}, x_{2} \geq 0$ with parameters $c_{2}, c_{3}, \omega>0$. One can verify that $r_{X_{1}}\left(x_{1}\right)$ is a decreasing and $r_{X_{2}}\left(x_{2}\right)$ is an increasing function, with $r_{X_{1}}(0)=1$ and $r_{X_{2}}(0)=\omega c_{3}$. So, the upper bound for $\theta$ from (4.10) is $\omega\left(c_{3}+1\right)$.

Inequalities (4.9a) and (4.9b) imply that $\theta \geq 2 \omega$ and $\theta \geq \frac{\omega\left(c_{3}^{2}-2 c_{2}\right)}{c_{3}}$. Therefore, $\tau=\frac{\omega\left(c_{3}^{2}-2 c_{2}\right)}{c_{3}}$ when $\frac{\left(c_{3}^{2}-2 c_{2}\right)}{c_{3}} \leq 2$ and we obtain the following additional restrictions of parameters: $2 c_{2} \geq c_{3}\left(c_{3}-2\right)$ and $c_{3} \geq 2$. Thus, the admissible values of $\theta$ belong to the interval $\left[\max \left(\frac{\omega\left(c_{3}^{2}-2 c_{2}\right)}{c_{3}}, \omega c_{3}\right), \omega\left(1+c_{3}\right)\right]$.

With the help of (3.3) we arrive to the following valid survival function

$$
S\left(x_{1}, x_{2}\right)=\left\{\begin{array}{l}
\frac{1}{1+x_{1}-\omega x_{2}} \exp \left\{-\theta x_{2}\right\} \\
\quad \text { if } x_{1} \geq \omega x_{2}, \\
\exp \left\{-\left[\left(\frac{\theta}{\omega}-c_{3}\right) x_{1}+c_{2}\left(x_{1}^{2}+\omega^{2} x_{2}^{2}\right)+\omega x_{2}\left(c_{3}-2 c_{2} x_{1}\right)\right]\right\}, \\
\quad \text { if } x_{1} \leq \omega x_{2} .
\end{array}\right.
$$

Finally, if $\theta=\omega\left(c_{3}+1\right)$ the joint distribution is absolutely continuous.

On Figure 9 we show the shape of the joint density when it has a singular component and in absolutely continuous case (the singularity line $x_{1}=1.2 x_{2}$ is given in red), see graphs (a) and (b), correspondingly.

4.2.2 Distributions with independent marginals. Let the marginals $X_{1}$ and $X_{2}$ of continuous joint distribution $\left(X_{1}, X_{2}\right)$ be independent. In terms of conditional 
(a)

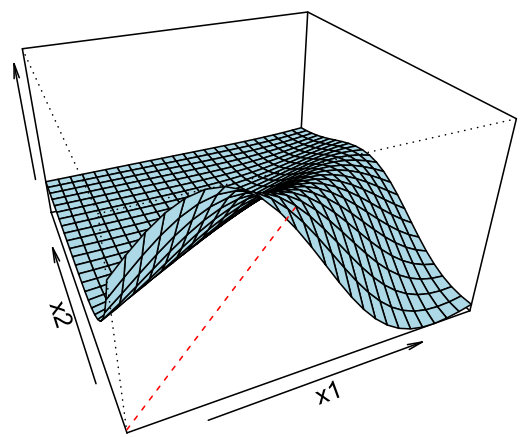

(b)

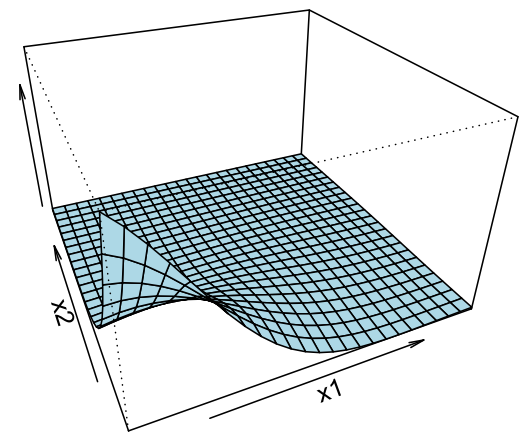

Figure 9 Density $f_{X_{1}, X_{2}}\left(x_{1}, x_{2}\right)$ in Example 4.4 for $c_{2}=4.0$ and $c_{3}=5.0$. (a) $\omega=1.2$ and $\theta=5.0$ (with singular part); (b) $\omega=1.2$ and $\theta=6.0$ (absolutely continuous).

failure rates, the independence between $X_{1}$ and $X_{2}$ implies that

$$
r_{i}\left(x_{1}, x_{2}\right)=\frac{\partial}{\partial x_{i}}\left[-\ln S_{X_{1}, X_{2}}\left(x_{1}, x_{2}\right)\right]=r_{X_{i}}\left(x_{i}\right), \quad i=1,2,
$$

i.e., $r_{1}\left(x_{1}, x_{2}\right)+r_{2}\left(x_{1}, x_{2}\right)=r_{X_{1}}\left(x_{1}\right)+r_{X_{2}}\left(x_{2}\right)$. Assume further that $\left(X_{1}, X_{2}\right)$ possesses W-BLMP, for example, $S_{X_{1}, X_{2}}\left(x_{1}, x_{2}\right)$ is given by (3.3) and we obtain

$$
r_{X_{1}}\left(x_{1}\right)+r_{X_{2}}\left(x_{2}\right)= \begin{cases}\theta+(1-\omega) r_{X_{1}}\left(x_{1}-\omega x_{2}\right), & \text { if } x_{1} \geq \omega x_{2}, \\ \frac{\theta}{\omega}+\left(1-\frac{1}{\omega}\right) r_{X_{2}}\left(x_{2}-\frac{x_{1}}{\omega}\right), & \text { if } x_{1} \leq \omega x_{2} .\end{cases}
$$

Let $x_{1} \geq \omega x_{2}$. Then equation

$$
r_{X_{1}}\left(x_{1}\right)+r_{X_{2}}\left(x_{2}\right)=\theta+(1-\omega) r_{X_{1}}\left(x_{1}-\omega x_{2}\right)
$$

is valid for all $x_{1}, x_{2} \geq 0$ and $\omega>0$. Substitute $x_{2}=0$ in (4.13) to conclude that $\omega r_{X_{1}}\left(x_{1}\right)=\theta-r_{X_{2}}(0)$ for all $x_{1} \geq 0$. Thus, the failure rate $r_{X_{1}}\left(x_{1}\right)$ is a positive constant $\frac{\theta-r_{X_{2}}(0)}{\omega}$ for all $x_{1} \geq 0$ and therefore $X_{1}$ is exponentially distributed with a parameter $\frac{\theta-r_{X_{2}}(0)}{\omega}$.

Now set $x_{1}=x_{2}=0$ in (4.13) to get $\theta=\omega r_{X_{1}}(0)+r_{X_{2}}(0)$, which is the upper bound in (4.11), meaning that the corresponding joint distribution is absolutely continuous according to Lemma 4.1 .

By analogy, if $x_{1} \leq \omega x_{2}$ we obtain that $X_{2}$ is exponentially distributed with a parameter $\theta-\omega r_{X_{1}}(0)$. Similarly, the corresponding joint distribution should be absolutely continuous. Hence, we proved the following.

Corollary 4.2. If a joint distribution $\left(X_{1}, X_{2}\right)$ with independent marginals possesses $W$-BLMP, then it is absolutely continuous. Moreover, the marginals $X_{1}$ and $X_{2}$ are exponentially distributed. 
The proof of Corollary 4.2 indicates that if $X_{1}$ has an exponential distribution with a parameter $\lambda_{1}>0$, then $X_{2}$ is exponentially distributed with a parameter $\lambda_{2}=\theta-\omega \lambda_{1}>0$. Thus, the corresponding joint absolutely continuous distribution with independent marginals is given by

$$
S_{X_{1}, X_{2}}\left(x_{1}, x_{2}\right)=\exp \left\{-\lambda_{1} x_{1}-\left(\theta-\omega \lambda_{1}\right) x_{2}\right\}
$$

4.2.3 Closure properties. Let $\mathcal{W}\left(x_{1}, x_{2} ; \Theta\right)$ be the collection of proper bivariate distributions possessing W-BLMP and their marginals satisfying Theorem 4.2. Denote by $\Theta=(\theta, \omega, \Lambda)$ the corresponding (generic) parameter vector, with $\Lambda$ being the vector of all parameters of marginal distributions. Note that $\Theta$ will be different under update of parameters $\theta, \omega$ or $\Lambda$. We will present in the next statement several closure properties of the class $\mathcal{W}\left(x_{1}, x_{2} ; \Theta\right)$.

Theorem 4.3. Denote by $\mathcal{S}, \mathcal{S}_{1}$ and $\mathcal{S}_{2}$ survival functions belonging to the class $\mathcal{W}\left(x_{1}, x_{2} ; \Theta\right)$. The following closure properties are fulfilled:

(CP1) If $\mathcal{S}_{1}, \mathcal{S}_{2} \in \mathcal{W}\left(x_{1}, x_{2} ; \Theta\right)$, then their product $\mathcal{S}_{1} \mathcal{S}_{2} \in \mathcal{W}\left(x_{1}, x_{2} ; \Theta\right)$.

(CP2) If $\mathcal{S} \in \mathcal{W}\left(x_{1}, x_{2} ; \Theta\right)$, then $[\mathcal{S}]^{c} \in \mathcal{W}\left(x_{1}, x_{2} ; \Theta\right)$ for some $c \geq 1$.

(CP3) If $\mathcal{S}_{1}, \mathcal{S}_{2} \in \mathcal{W}\left(x_{1}, x_{2} ; \Theta\right)$, then $\left[\mathcal{S}_{1}\right]^{c_{1}}\left[\mathcal{S}_{2}\right]^{c_{2}} \in \mathcal{W}\left(x_{1}, x_{2} ; \Theta\right)$ for some $c_{1}, c_{2} \geq 1$.

(CP4) If $S_{Y_{1}, Y_{2}}\left(x_{1}, x_{2}\right)$ belongs to $\mathcal{W}\left(x_{1}, x_{2} ; \Theta\right)$ and $\beta>0$, then $S_{X_{1}, X_{2}}\left(x_{1}, x_{2}\right)=$ $S_{Y_{1}, Y_{2}}\left(\beta x_{1}, \beta x_{2}\right)$ also belongs to $\mathcal{W}\left(x_{1}, x_{2} ; \Theta\right)$.

(CP5) If $\mathcal{S}_{1}, \mathcal{S}_{2}$ have the same parameter vector $\Theta$ and belong to $\mathcal{W}\left(x_{1}, x_{2} ; \Theta\right)$, then $\frac{\mathcal{S}_{1}+\mathcal{S}_{2}}{2} \in \mathcal{W}\left(x_{1}, x_{2} ; \Theta\right)$.

Proof. The properties (CP1) to (CP5) follow after applying relation (3.3) for the corresponding case.

Remark 4.2 (Applications of closure properties). The closure properties listed in Theorem 4.3 enable us to construct plenty of bivariate distributions belonging to $\mathcal{W}\left(x_{1}, x_{2} ; \Theta\right)$ by using as a base a known distribution defined by (3.3), being an output of Theorem 4.2. Moreover, relations (CP1), (CP3) and (CP5) can be easily extended to any finite number of survival functions belonging to $\mathcal{W}\left(x_{1}, x_{2} ; \Theta\right)$.

Note that if the vector $\left(Y_{1}, Y_{2}\right)$ is independent of the vector $\left(Z_{1}, Z_{2}\right)$ and their survival functions belong to $\mathcal{W}\left(x_{1}, x_{2} ; \Theta\right)$ class, the implication $(\mathrm{CP} 1)$ means that the survival function of the vector

$$
\left(X_{1}, X_{2}\right)=\left[\min \left(Y_{1}, Z_{1}\right), \min \left(Y_{2}, Z_{2}\right)\right]
$$

also belongs to $\mathcal{W}\left(x_{1}, x_{2} ; \Theta\right)$. This fact has applications in series systems.

We finish with a complementary closure property given in the following claim. 
Lemma 4.3. If $S_{T_{1}, T_{2}}\left(x_{1}, x_{2}\right)$ belongs to $\mathcal{W}\left(x_{1}, x_{2} ; \Theta\right)$ and $T_{3}$ is independent of $\left(T_{1}, T_{2}\right)$ with a survival function

$$
S_{T_{3}}\left(\max \left(x_{1}, \omega x_{2}\right)\right)= \begin{cases}\exp \left\{-\lambda \omega x_{2}\right\}, & \text { if } x_{1} \geq \omega x_{2}, \\ \exp \left\{-\lambda x_{1}\right\}, & \text { if } x_{1} \leq \omega x_{2},\end{cases}
$$

for all $x_{1}, x_{2} \geq 0$ and $\lambda>0$, then stochastic relation (2.1) generates a joint distribution belonging to the class $\mathcal{W}\left(x_{1}, x_{2} ; \Theta\right)$.

Proof. Let $S_{T_{1}, T_{2}}\left(x_{1}, x_{2}\right)$ be specified by (3.3), for example, our base class is $\mathcal{W}\left(x_{1}, x_{2} ; \Theta\right)$ with $\Theta=(\theta, \omega, \Lambda)$. Using stochastic representation (2.1) we obtain

$$
S_{X_{1}, X_{2}}\left(x_{1}, x_{2}\right)=S_{T_{1}, T_{2}}\left(x_{1}, x_{2}\right) S_{T_{3}}\left(\max \left(x_{1}, \omega x_{2}\right)\right)
$$

and applying (4.15) we get

$$
S_{X_{1}, X_{2}}\left(x_{1}, x_{2}\right)= \begin{cases}\exp \left\{-(\theta+\lambda \omega) x_{2}\right\} S_{X_{1}}\left(x_{1}-\omega x_{2}\right), & \text { if } x_{1} \geq \omega x_{2}, \\ \exp \left\{-\frac{(\theta+\lambda \omega) x_{1}}{\omega}\right\} S_{X_{2}}\left(x_{2}-\frac{x_{1}}{\omega}\right), & \text { if } x_{1} \leq \omega x_{2} .\end{cases}
$$

Therefore, we remain in the class $\mathcal{W}\left(x_{1}, x_{2} ; \Theta\right)$, but now $\Theta=\left(\theta_{1}, \omega, \Lambda\right)$ with $\theta_{1}=\theta+\lambda \omega$.

Observe that distribution (4.15) exhibits a cusp along the line $L_{\omega}=\left\{x_{1}=\omega x_{2}\right\}$, as shown on Figure 10, representing the corresponding "level curve" for fixed $x_{2}=$ $x_{0}=1.8$, when $\omega=0.5$ and $\lambda=1.0$.

Recall that Pinto and Kolev (2015) introduced an Extended MO model with singularity along the line $\left\{x_{1}=x_{2}\right\}$ in $R_{+}^{2}$. The important consequence of Lemma 4.3 is that it gives us an idea how to define a modified Extended MO model, but with a possible singularity along the line $L_{\omega}:\left\{x_{1}=\omega x_{2}\right\}$.

\section{Discussion and possible further investigations}

The message of this work is to introduce a weak version of the classical BLMP property, that we named W-BLMP, via functional equation (1.5) generated by the stochastic representation (2.1). The additional parameter $\omega$ is the inclination of arbitrary line in $R_{+}^{2}$ passing through the origin. The examples illustrating our approach indicate that the asymmetry parameter $\omega$ plays a role of a skew parameter and implies a delayed effect of the common shock affecting one of the items of a system. As an interpretation, the W-BLMP preserves the distribution of random vector $\left(X_{1}, X_{2}\right)$ considering its dynamic in direction of the line $L_{\omega}:\left\{x_{2}=\omega x_{1}\right\}$, instead of keeping it unchanged along the main diagonal, as postulated by BLMP.

The W-BLMP is characterized by the special form of joint survival function $S_{X_{1}, X_{2}}\left(x_{1}, x_{2}\right)$ given by (3.3) which is a solution of functional equation (1.5). This 


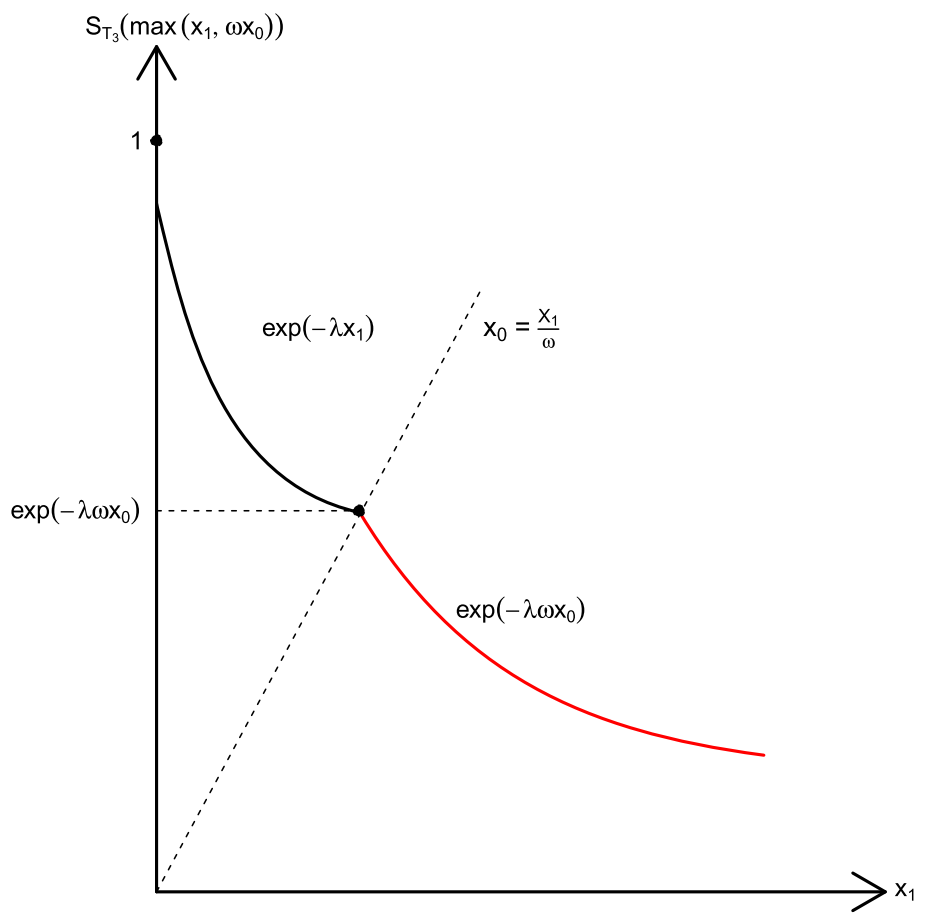

Figure 10 Shape of $S_{T_{3}}\left(\min \left(x_{1}, \omega x_{2}\right)\right)$ for $\omega=0.5, \lambda=1.0$ and $x_{0}=1.8$.

fact is a contribution to the theory of functional equations. The proof of Theorem 3.1 is different from existing approaches treating the BLMP case. We use the fact that the independent solution (3.2) of functional equation (3.1) may serve as a particular solution of (1.5). Finally, we suggest an algorithm for building distributions possessing W-BLMP starting from pre-specified marginal distributions that must satisfy a list of restrictions and enrich it by closure properties of the new BLMP notion.

We will list below several related research problems.

- One would be successful to use our methodology when the support of the singular component lies along a monotonic curve different than the line through the origin. The choice of the model should be influenced on the data at hand.

- Of course, depending on the situation, one might consider a dual version of the W-BLMP replacing the min by max operation in stochastic representation (2.1). Now, the interest will be related to model our data by the joint distribution function of $\left(X_{1}, X_{2}\right)$ instead of joint survival one (when we have right censoring, for instance).

- Practical needs indicate that it makes sense to define models with restricted domain, but not "for all $x_{1}, x_{2} \geq 0$ " as one usually assumes as a standard. For example, one can still use as a base the functional equation (1.5), but when its 
arguments belong to a fixed figure, indicated by corresponding scatterplot, a square say. In such a case we will deal with a weaker version of the W-BLMP.

- The "delayed" effect might be identified in the form of a fixed time interval, say " $d$ ", instead of the multiplicative factor $\omega$ assumed in this paper. Of course, one could start with a fixed value of " $d$ " and, in a second step, to plug-in " $d$ " as a parameter of the joint distribution, as we did with the parameter $\omega$. Such kind of delayed effect would be graphically represented as a shift (upwards or downwards) of the usual support of the singular component, keeping the 45 degree inclination.

- We see promising application of Sibuya's dependence function, being the ratio of joint distribution and the product of marginal distributions, see more details in Pinto and Kolev (2016). The idea is to investigate under which conditions the Sibuya's dependence function is invariant along the line $L_{\omega}$, that is, when the Sibuya's dependence function of $\left(X_{1}, X_{2}\right)$ and its $\omega$-type residual lifetime vector $\mathbf{X}_{w, t}$ are the same for all $t>0$.

- In Lemma 4.3, we got a modification of extended Marshall-Olkin distribution given by (4.16) which is based on stochastic representation (2.1). The dependence structure between two items of a system is explained not only by the common shock identified by the random variable $T_{3}$, but also by the joint distribution of the individual shocks represented by $T_{1}$ and $T_{2}$. With this additional source of dependence extended Marshall-Olkin's distributions allow modeling both positive and negative quadrant dependence between its components. Moreover, the model may be non-exchangeable even if the marginals have the same distribution, consult Pinto and Kolev (2015) for details.

The classical MO model (1.2) is probably one of the mostly used in Reliability, Survival analysis and Life Insurance. Hence, any extension of that model has its theoretical and practical importance. We expect that the modified MO models introduced in this article may serve as an alternative to existing ones, with a significant effect on analysis of specific data sets.

\section{Acknowledgments}

We are grateful to the referees whose remarks significantly improved the above exposition. We are thankful to Hugo Brango who designed the figures. The first author is partially supported by FAPESP grant 2013/07375-0.

\section{References}

Aczél, J. (1966). Lectures on Functional Equations and Their Applications. New York: Academic Press. MR0208210

Balakrishnan, N. and Lai, C.-D. (2009). Continuous Bivariate Distributions, 2nd ed. New York: Springer. MR2840643 
Barlow, R. and Proschan, F. (1981). Statistical Theory of Reliability and Life Testing. Silver Spring: To Begin With. MR0438625

Block, H. W. and Basu, A. P. (1974). A continuous bivariate exponential extension. Journal of the American Statistical Association 69, 1031-1037. MR0433713

Brigo, D., Mai, J.-F. and Scherer, M. (2016). Markov multi-state survival indicators for default simulation as a new characterization of the Marshall-Olkin law. Statistics \& Probability Letters 114, 60-66. MR3491973

Cherubini, U., Durante, F. and Mulinacci, S. (2015). Marshall-Olkin Distributions-Advances in Theory and Applications. Springer Series in Mathematics \& Statistics 141. Cham: Springer. MR3368565

Esary, J. and Marshall, A. (1974). Multivariate distributions with exponential minimums. The Annals of Statistics 2, 84-98. MR0362704

Friday, D. S. and Patil, G. P. (1977). A bivariate exponential model with applications to reliability and computer generation of random variables. In The Theory and Applications of Reliability (C. P. Tsokos and I. N. Shimi, eds.) 527-549. New York: Academic Press. MR0519107

Galambos, J. and Kotz, S. (1978). Characterizations of Probability Distributions. Lecture Notes in Mathematics 675. Berlin: Springer. MR0513423

Gupta, A., Zeung, W. and Hu, Y. (2010). Probability and Statistical Models: Foundations for Problems in Reliability and Financial Mathematics. New York: Birkhauser. MR2680605

Joe, H. (2015). Dependence Modeling with Copulas. Boca Raton: CRC Press. MR3328438

Johnson, N. L. and Kotz, S. (1975). A vector multivariate hazard rate. Journal of Multivariate Analysis 5, 53-66. MR0365901

Kulkarni, H. V. (2006). Characterizations and modelling of multivariate lack of memory property. Metrika 64, 167-180. MR2259220

Lancaster, H. (1957). Some properties of the bivariate normal distribution considered in the form of a contingency table. Biometrika 44, 289-292.

Li, X. and Pellerey, F. (2011). Generalized Marshall-Olkin distributions and related bivariate aging properties. Journal of Multivariate Analysis 102, 1399-1409. MR2819957

Liebscher, E. (2008). Construction of asymmetric multivariate copulas. Journal of Multivariate Analysis 99, 2234-2250. MR2463386

Lin, G., Lai, C.-D. and Govindaraju, K. (2016). Correlation structure of the Marshall-Olkin bivariate exponential distribution. Statistical Methodology 29, 1-9. MR3429151

Marshall, A. (1975). Some comments on the hazard gradient. Stochastic Processes and Their Applications 3, 293-300. MR0400600

Marshall, A. and Olkin, I. (1967). A multivariate exponential distribution. Journal of the American Statistical Association 62, 30-44. MR0215400

Marshall, A. and Olkin, I. (1991). Functional equations for multivariate exponential distributions. Journal of Multivariate Analysis 39, 209-215. MR1128681

Marshall, A. and Shaked, M. (1979). Multivariate shock models for distributions with increasing hazard rate average. Annals of Probability 7, 343-358. MR0525058

McNeil, A., Frey, L. and Embrechts, P. (2015). Quantitative Risk Management, 2nd ed. Princeton: Princeton University Press. MR3445371

Okyere, E. (2007). Maximum likelihood analysis for bivariate exponential distributions. Ph.D. thesis, University of Gottingen.

Pinto, J. and Kolev, N. (2015). Extended Marshall-Olkin model and its dual version. In MarshallOlkin Distributions-Advances in Theory and Applications (U. Cherubini, F. Durante and S. Mulinacci, eds.). Springer Series in Mathematics \& Statistics 141 87-113. Cham: Springer. MR3368566

Pinto, J. and Kolev, N. (2016). A class of continuous bivariate distributions with linear sum of hazard gradient components. Journal of Statistical Distributions and Applications 3, 1-17. 
Proschan, F. and Sullo, P. (1974). Estimating the parameters of a bivariate exponential distribution in several sampling situations. In Reliability and Biometry: Statistical Analysis of Life Lengths (F. Proschan and R. J. Serfling, eds.) 423-440. MR0348901

Singpurwalla, N. (2006). Reliability and Risk: A Bayesian Perspective. Chichester: Wiley. MR2265917

\author{
Department of Statistics \\ University of São Paulo \\ Rua do Matão 1010 \\ 05508-090 São Paulo \\ Brazil \\ E-mail: kolev.ime@gmail.com \\ jaymeaugusto@gmail.com
}

